\title{
Algebraic Signal Processing Theory: Sampling for Infinite and Finite 1-D Space
}

\author{
Jelena Kovačević, Fellow, IEEE and Markus Püschel, Senior Member, IEEE
}

\begin{abstract}
We derive a signal processing framework, called space signal processing, that parallels time signal processing. As such, it comes in four versions (continuous/discrete, infinite/finite), each with its own notion of convolution and Fourier transform. As in time, these versions are connected by sampling theorems that we derive. In contrast to time, however, space signal processing is based on and derived from a different notion of shift, space shift, which operates symmetrically. Our work rigorously connects known and novel concepts into a coherent framework; most importantly, it shows that the sixteen discrete cosine and sine transforms are the space equivalent of the discrete Fourier transform, and hence can be derived by sampling. The platform for our work is the algebraic signal processing theory, an axiomatic approach and generalization of linear signal processing that we recently introduced.
\end{abstract}

\section{INTRODUCTION}

The theory of time signal processing in one dimension is the foundation of our discipline. It consists of four closely related variants depending on the nature of the time domain (see Fig. 1): infinite continuous, finite (meaning finite duration) continuous and periodically extended, infinite discrete, and finite discrete and periodically extended. Each case has its own notion of filtering or convolution, spectrum, and Fourier transform. For example, infinite discrete-time signal processing has the discrete-time Fourier transform (DTFT) as Fourier transform and the spectrum is periodic, that is, continuous, finite, and periodically extended (see Fig. 1). Note that all visualizations in Fig. 1 are directed, representing the directed flow of time formally captured by the time shift discussed next.

The time signal processing framework for these four cases can be systematically derived from one basic concept: the continuous-time shift. Assume a signal $x(t)$ on $\mathbb{R}$. For some $T \in \mathbb{R}$, the time shift is defined as follows:

$$
\text { time shift by } T: \quad x(t) \rightarrow x(t-T) .
$$

Convolution can be viewed as a superposition of weighted shifts and is defined as

$$
h(t) * x(t)=\int h(\tau) x(t-\tau) d \tau .
$$

The spectrum consists of the simultaneous eigenfunctions $\exp (j \omega t), \omega \in \mathbb{R}$, for this convolution, and the Fourier

This work was supported by NSF through awards 310941, 0634967, 0515152, 633775, 0331657, and by the PA State Tobacco Settlement, KamletSmith Bioinformatics Grant.

J. Kovačević is with the Department of Biomedical Eningeering and the Department of Electrical and Computer Engineering, Carnegie Mellon University, Pittsburgh. E-mail: jelenak@cmu.edu. M. Püschel is with the Department of Electrical and Computer Engineering, Carnegie Mellon University, Pittsburgh. E-mail: pueschel@ece.cmu.edu.

Parts of this work were presented in [1]. transform is defined as the projection onto these eigenfunctions. In summary, we obtain the basic framework for infinite continuous-time signal processing (Fig. 1, top left).

The other three cases of time signal processing can be derived through equidistant sampling. Sampling in the time domain yields infinite discrete-time signal processing, sampling in the frequency domain yields the finite continuous-time signal processing, and sampling in both domains yields finite discrete-time signal processing. The Fourier transform for the latter is called the discrete Fourier transform (DFT), one of the most important tools in time signal processing; sampling is one way to derive the DFT.

Another important class of transforms used in signal processing are the discrete cosine and sine transforms (DCTs/DSTs), originally derived and thought of as approximations to the Karhunen-Loève transform of a first-order GaussMarkov process [2], [3].

In this paper, we ask the question: Can a signal processing framework analogous to time signal processing in Fig. 1 be derived such that the DCTs/DSTs are obtained similarly to the DFT? As we will show, the answer is positive. As an example, a visualization of the framework underlying the most widely used trigonometric transform, DCT type 2, is shown in Fig. 2. The details will be explained later; here, we only want to point out two major differences with respect to time signal processing. First, the visualizations are now undirected (no inherent notion of past and future) and hence we refer to this framework as space signal processing. Second, in time signal processing, the four structures in the time domain are equal to the four structures in the frequency domain. In space signal processing this is not the case unless all sixteen DCTs/DSTs are considered jointly.

Based on the above discussion of time signal processing, it becomes clear that space signal processing, if it exists, has to be based on a different notion of shift. Indeed, the solution is what we call the space shift, which operates undirected, symmetrically to the left and the right:

$$
\text { space shift by } T: \quad x(t) \rightarrow \frac{1}{2}(x(t+T)+x(t-T)) .
$$

The associated notion of convolution is now obtained as superposition of space shifts:

$$
h(t) *_{S / A} x(t)=\int h(\tau) \frac{1}{2}(x(t+\tau)+x(t-\tau)) d \tau,
$$

where $*_{S / A}$ denotes this new convolution, which comes in two flavors: $\mathrm{S}$ and A. After defining these, we derive the necessary sampling theorems to obtain the complete framework partially shown in Fig. 2. We achieve here several goals: 

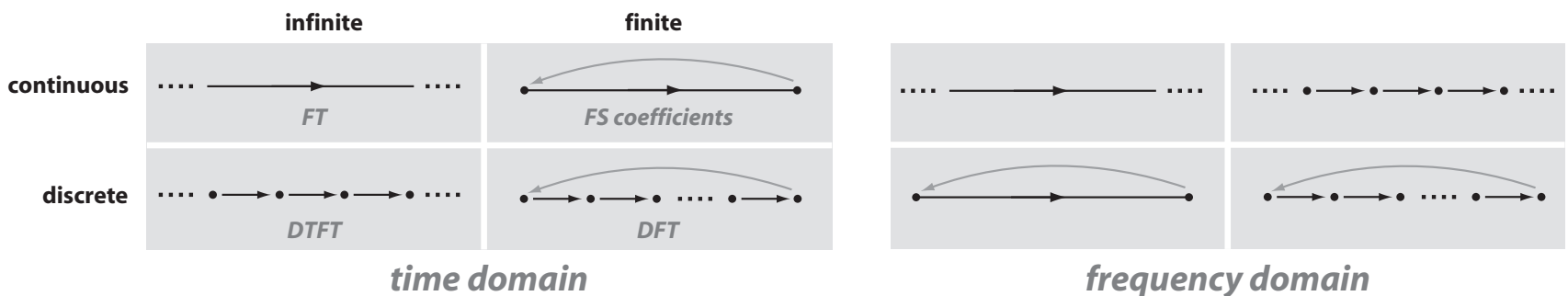

frequency domain

Fig. 1. Four variants of time signal processing. Left: the structures of the time domains; right: the structures of the corresponding Fourier domains. The backwards arrow signifies a periodic signal extension beyond the shown signal domain (interval).
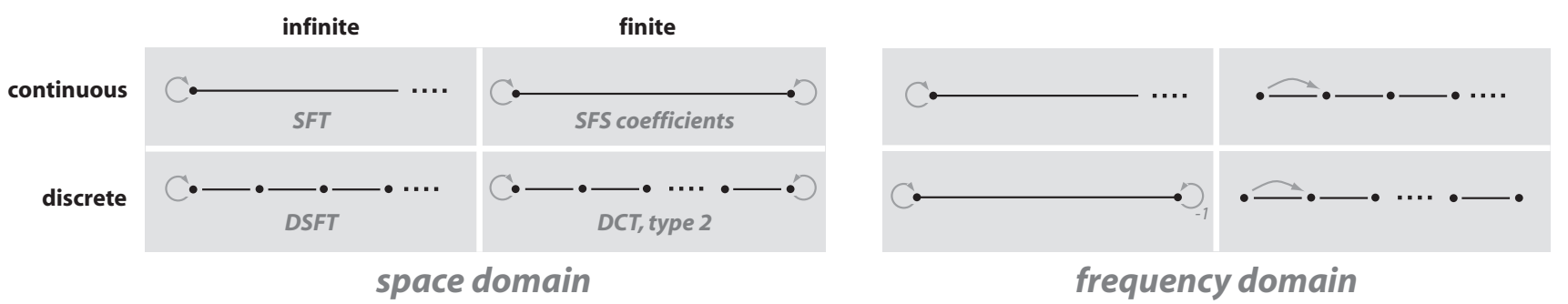

Fig. 2. Four variants of space signal processing. Left: the structures of the space domains; right: the structures of the corresponding Fourier domains. The first S in SFT, SFS, and DSFT stands for "space." The looping arrows signify different forms of symmetric and antisymmetric signal extensions. Details are introduced later.

- we demonstrate that the DFT and DCTs/DSTs are equivalent concepts with an equivalent underlying framework in the most rigorous sense;

- we provide a novel way of deriving the DCTs/DSTs; and,

- we show that a complete linear signal processing framework different from standard time signal processing can be derived.

The platform for our work is the algebraic signal processing theory (ASP), a general axiomatic approach to signal processing built on the basic concept of a signal model [4], [5]. For example, the four cases in Fig. 1 are different signal models. We showed already how to derive discrete-time and discretespace signal models (in the sense used here) from the shift definition "bottom-up," that is, without the use of continuous signal processing and sampling [4], [6]. Here we complete the picture by showing the appropriate continuous-space models and deriving sampling theorems. Our approach in this paper is meant to be general enough so as to be applicable to other forms of nontime signal processing, such as 2-D hexagonal space [7], 2-D quincunx space [8], or others.

Related work. Our work is closely related to both Fourier analysis on symmetric or antisymmetric ${ }^{1}$ signals and prior work that establishes the close relationship between DCTs/DSTs and the DFT of various symmetric/antisymmetric signals. For example, the Fourier cosine transform [3] is associated with symmetric infinite continuous signals and periodic functions with symmetries have Fourier series of a particular form [9], [10]. Further, DCTs/DSTs are closely related to a DFT on a signal with symmetry [11], [12] and have special associated convolutions. Along similar lines, continuous equivalents of the discrete cosine transform were defined in [13], [14], [15], [16] to perform interpolation using zero-padding in the frequency domain (similar to DFT-based interpolation).

\footnotetext{
${ }^{1}$ Sometimes called even and odd.
}

Our work ties these concepts, and others that we define, into one coherent framework that precisely parallels time signal processing and that is held together by sampling theorems. Unser in [17] discusses the modern work in sampling theory, including generalizations which, instead of using bandlimited spaces spanned by sincs, use other approximation spaces, spanned by splines and wavelets, for example.

Organization of the paper. In Section II, we start by identifying the general definitions of signals, filters, and filtering (convolution) within the algebraic signal processing theory. We then use these generic concepts to present infinite continuousspace signal processing in Section III and derive its sampling theorems in Section IV. The frequency domains of the so obtained discrete-space models give rise to four finite continuousspace models that we define in Section V. Sampling those in Section VI finally yields the sixteen DCTs/DSTs as Fourier transforms for finite discrete space models.

\section{Algebraic Signal Processing Theory}

We start with a short overview of the ASP introduced in [4], [5], [6]. ASP is a general and axiomatic approach to linear signal processing (henceforth simply called signal processing). For this paper, ASP provides the natural platform for the introduction of space signal processing and for the organization of signal processing concepts to clearly identify parallels and differences.

Algebraic signal processing theory: Overview. Two key observations underlie ASP: the algebraic nature of signal processing and the concept of a signal model, a collection of three objects sufficient to define a signal processing framework.

The set of filters in signal processing is usually assumed to be a vector space (addition $=$ parallel connection, scalar multiplication $=$ amplification $)$, but also offers multiplication (serial connection), an operation outside the vector space 
framework. Thus, algebraically, the filter space is more than a vector space: it is an algebra $\mathcal{H}$, a vector space that is also a ring. Further, the set of signals is also typically assumed to be a vector space, and filters operate on this space via filtering. Algebraically, this means that the signal space is an $\mathcal{H}$-module $\mathcal{S}$. These observations naturally place signal processing into the context of representation theory of algebras, a welldeveloped mathematical discipline (see [18], for example).

ASP is axiomatically built on top of the concept of a signal model, defined as a triple $(\mathcal{H}, \mathcal{S}, \Phi)$, where $\mathcal{H}$ is a chosen filter algebra, $\mathcal{S}$ an associated $\mathcal{H}$-module of signals, and $\Phi$ a bijective mapping from a vector space $V$ into the module $\mathcal{S}$. The purpose of $\Phi$ is to assign a module and an algebra to a signal (vector) space, so filtering and other concepts are defined. ASP now asserts: If a signal model $(\mathcal{H}, \mathcal{S}, \Phi)$ is given, all the basic ingredients for signal processing are automatically defined (if they exist) and provided by the representation theory of algebra. These basic ingredients include the notions of filtering, spectrum, and Fourier transform, and others, and usually take different forms for different signal models.

Example: Time signal processing. In ASP, each of the four variants of time signal processing in Fig. 1 is a signal model for a different vector space $V$. For example, in the infinite continuous-time model, $V$ is typically assumed to be the space of finite energy functions $V=L^{2}(\mathbb{R})$. The signal module $\mathcal{S}$ is identical to $V$ as a set, but not as an algebraic structure: it possesses an algebra operating on it, typically chosen as ${ }^{2}$ $\mathcal{H}=L^{1}(\mathbb{R}) . \Phi$ is the identity mapping from $V$ to $\mathcal{S}$. The Signal model concepts for the infinite continuous-time model are summarized in Table I.

As discussed in the introduction, the infinite continuoustime model is based on the time shift (1). The visualization of the time model in Table I shows the domain on which time signals live; the domain is directed due to the operation of the directed time shift.

From the definition of the signal model, Basic concepts follow, including the multiplication defined in the filter algebra (cascade of filters), and how filters operate on signals (in both cases standard convolution). In Spectral concepts, we define spectral components $\mathcal{S}_{\omega}$, which are nothing but subspaces of the signal module, invariant under the operation of the filter algebra (in signal processing, we typically find these by finding eigenfunctions). The Fourier transform $(\mathrm{FT})^{3}$ computes projections onto those subspaces. The spectral-domain visualization possesses the same structure as the time-domain one.

We then proceed to define the inverse FT, as well as the frequency response, found by filtering the spectral components and recording the eigenvalues. All this is well-known for the infinite continuous-time model. The benefits of the concept of a signal model and the general ASP framework become evident later when we consider different signal models.

We now briefly discuss the finite continuous-time model, which helps understand the need for the mapping $\Phi$ in the signal model. The finite time model is used for signals and

\footnotetext{
${ }^{2}$ Note that choosing $L^{2}(\mathbb{R})$ destroys the algebra property: the convolution of two finite-energy functions is in general not finite energy.

${ }^{3}$ We denote by FT the Fourier transform corresponding to the infinite continuous-time model.
}

TABLE I

ESSENTIAL CONCEPTS FOR THE INFINITE CONTINUOUS TIME MODEL.

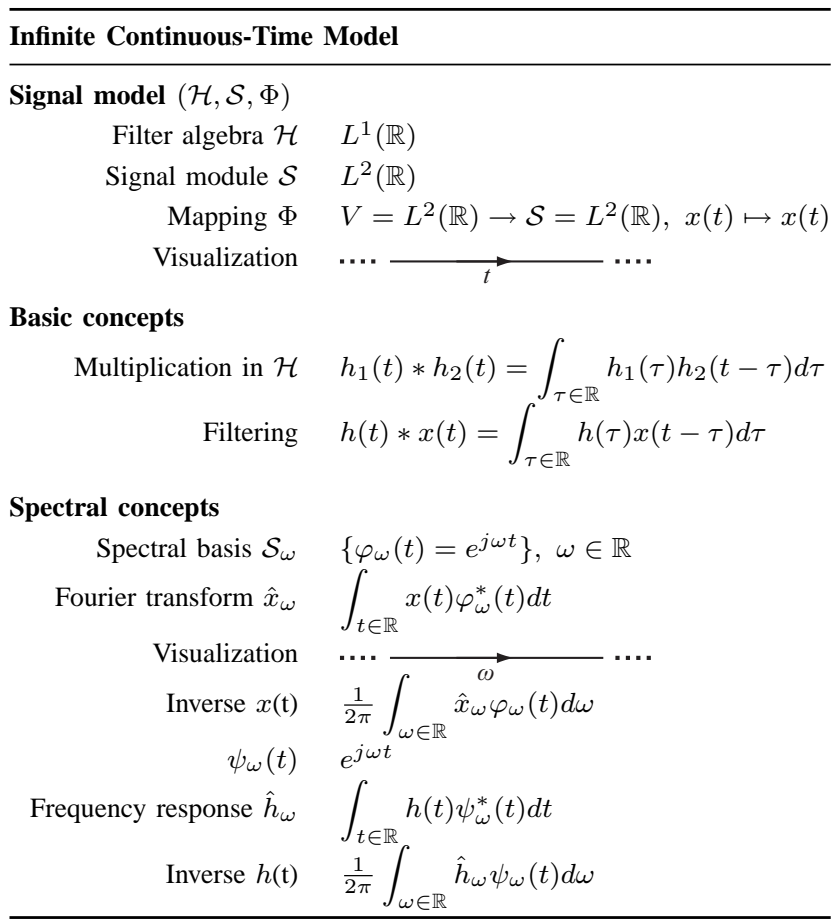

filters living on an interval, say, $\mathbb{I}=[0, I]$, and filtering is circular convolution $*_{P}(P$ stands for periodic $)$. Given $h(t), x(t)$ defined on $\mathbb{I}$, the circular convolution is computed by extending $x(t)$ periodically to $x_{P}(t)$, performing ordinary convolution, and viewing the (periodic) result as a function on II:

$$
h *_{P} x=h * x_{P} \quad \text { viewed as function on } \mathbb{I} .
$$

In ASP, we capture the required signal extension rigorously by viewing the signal domain as a circle $\mathbb{I}_{P}$ ( $P$ for periodically extended $\mathbb{I}$ ) with circumference $I$, instead of an interval. Intuitively, signals on the circle $\mathbb{I}_{P}$ are equivalent to periodic signals on the real line.

We summarize this model in Table II. Note that now we can choose $L^{1}$ for both filter and signal space. ${ }^{4}$ The purpose of $\Phi$ is again to assign a module and an algebra to a vector space. This time this includes "applying" the signal extension (by changing the domain of the signal from $\mathbb{I}$ to $\mathbb{I}_{P}$ ). The model is well-defined since the convolution in (5) yields again a periodic signal, that is, a signal on $\mathbb{I}_{P}$.

One may imagine that different signal extensions could be possible, which would require different mappings $\Phi$, as will indeed be the case later when we consider space models.

The Fourier transform for this model is again found by identifying the eigenspaces under filtering (now circular convolution). It is well known that these are spanned by complex exponentials: each

$$
\mathcal{S}_{k}=\left\{\varphi_{k}(t)=e^{j \frac{2 \pi k}{I} t}\right\}
$$

$k \in \mathbb{Z}$, is a simultaneous eigenspace for all filters $h$ in $\mathcal{H}$. The Fourier transform of a signal $x(t)$ is hence the projection onto

\footnotetext{
${ }^{4}$ Since the domain is compact, $L^{1}$ contains $L^{2}$.
} 
TABLE II

ESSENTIAL CONCEPTS FOR THE FINITE CONTINUOUS TIME MODEL.

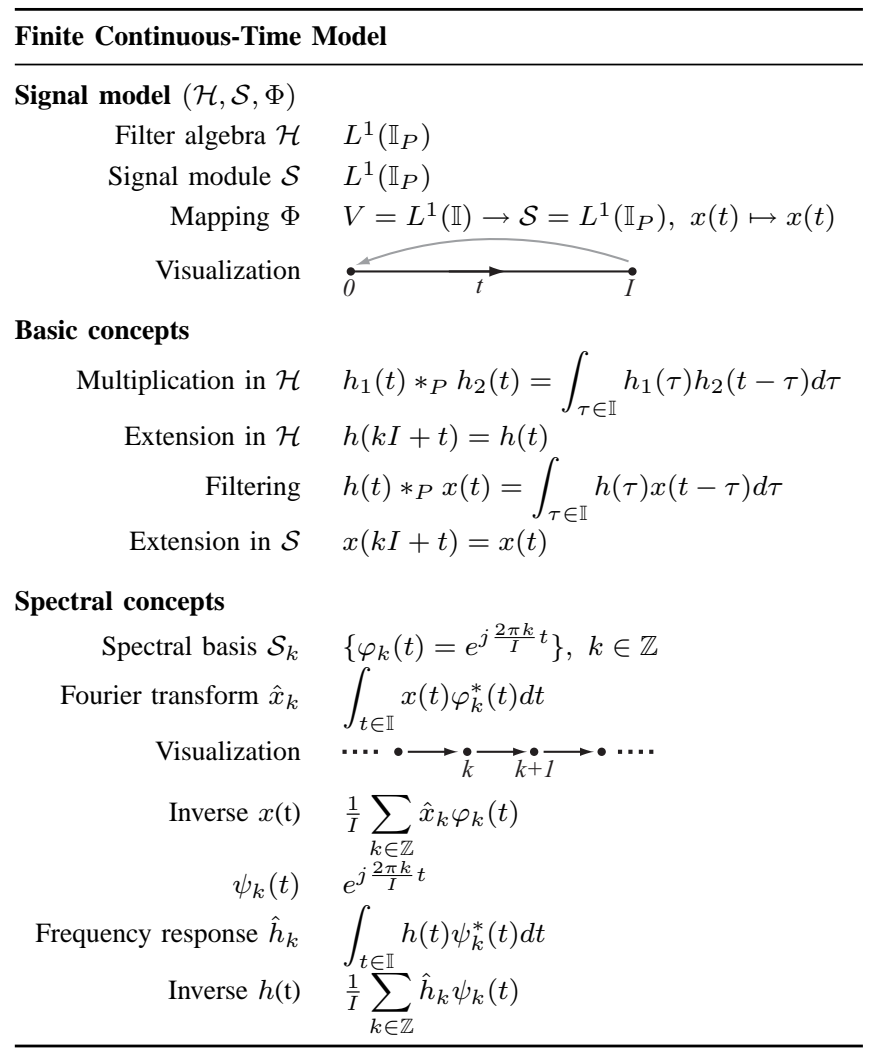

these spaces; it yields the coefficients of the Fourier series expansion of $x(t)$ (see Table II).

Structured signal domains through quotient sets. The proper framework to formally define the circle $\mathbb{I}_{P}$ and other structured signal domains needed later are equivalence relations and quotient sets [19]. While not strictly needed for understanding the paper, we briefly introduce this framework for completeness.

Intuitively, $\mathbb{I}_{P}$ is equal to the real line in which we identify all points that are at multiples of $I$ from each other. This way, the structure of a periodic signal on $\mathbb{R}$ is translated into the structure of the signal domain. The difference is a subtle one yet crucial in ASP. ${ }^{5}$

We briefly define equivalence relations and quotient sets in the simplest case using the above circle as example.

Let $S$ be a set. An equivalence relation $\sim$ on $S$ is a relation that satisfies three properties, namely for $a, b, c \in S$,

(1) $a \sim a, \quad(2) a \sim b \Rightarrow b \sim a, \quad(3) a \sim b \& b \sim c \Rightarrow a \sim c$.

For $a \in S,[a]=\{b \in S \mid b \sim a\}$ is called the equivalence class of $a$, and the set of all equivalence classes

$$
S / \sim=\{[a] \mid a \in S\}
$$

is the quotient set of $S$ with respect to $\sim$. The quotient set partitions $S$, that is, every $a \in S$ lies in exactly one equivalence class.

\footnotetext{
${ }^{5}$ For example, periodic signals viewed as functions on $\mathbb{R}$ have infinite energy and usually their power is computed. However, the power is just the energy if the signal is viewed as function on $\mathbb{I}_{P}$.
}

Connecting to the prior discussion, the equivalence relation $\sim_{P}$ on $S=\mathbb{R}$ defined as

$$
t_{1} \sim_{P} t_{2} \Leftrightarrow\left(t_{2}-t_{1}\right) / I \in \mathbb{Z}
$$

yields $\mathbb{R} / \sim_{P}=\mathbb{I}_{P}$. Namely, $\mathbb{I}=[0, I)$ is a complete set of pairwise nonequivalent points, and $I \sim_{P} 0$, which means the end points of $\mathbb{I}(0$ and $I)$ in Table II can be identified as shown by the backwards arrow of the visualization, creating the circle structure.

For some signal extensions (in this paper: antisymmetric) a more general version of the above construction is needed.

\section{InFINITE CONTINUOUS-SPACE Models}

We now formally define two infinite continuous-space models. These models will be related to the DCTs/DSTs in the same way as the infinite continuous-time model in Table $\mathrm{I}$ is related to the DFT.

As discussed in the introduction, these space models are obtained from a different notion of shift, the symmetric space shift (3), which is equivalent to convolving $x(t)$ with $(\delta(t-$ $T)+\delta(t+T)) / 2$. We define a space filter as superposition of space shifts as

$$
h(t)=\int h(\tau) \frac{1}{2}(\delta(t-T)+\delta(t+T)) d \tau .
$$

This implies that $h(t)=h(-t)$ is symmetric and hence we need to integrate over $\mathbb{R}^{+}$only. We view $h$ as a function on $\mathbb{R}^{+}$ symmetrically extended; formally, $h$ is a function on $\mathbb{R}_{S}^{+}=$ $\mathbb{R} / \sim_{S}$ with

$$
t_{1} \sim_{S} t_{2} \Leftrightarrow\left|t_{1}\right|=\left|t_{2}\right| .
$$

As signal space we could choose $L^{2}(\mathbb{R})$; however, to obtain the DCTs and DSTs through sampling, the right choice is to restrict to the signal space of symmetric and of antisymmetric signals (in our framework to signals on $\mathbb{R}_{S}^{+}$and $\mathbb{R}_{A}^{+}$, where $\mathbb{R}_{A}^{+}$denotes signals on $\mathbb{R}^{+}$, antisymmetrically extended). Note that if $s \in \mathbb{R}_{A}^{+}$, then necessarily $x(0)=0$.

Accordingly, we define in both cases space convolution or filtering $*_{S}, *_{A}$ as

$$
h(t) *_{S / A} x(t)=\int_{\tau \in \mathbb{R}^{+}} h(\tau) \frac{1}{2}(x(t+\tau)+x(t-\tau)) d \tau,
$$

where $x(t)$ is symmetrically or antisymmetrically extended (note that the extension of $x$ changes the actual computation in (8), hence the subscript S/A). We can also state this convolution in a form similar to (5). Namely, assume $h(t), x(t)$ are defined on $\mathbb{R}^{+}$, then

$$
h *_{S / A} x=\frac{1}{2}\left(h_{S} * x_{S / A}\right) \quad \text { viewed as function on } \mathbb{R}^{+} \text {. }
$$

Thus, to compute, say, $\mathrm{S}$ convolution, one first extends the signal symmetrically, computes the ordinary convolution, and then retains the part on $\mathbb{R}^{+}$. The factor $1 / 2$ simplifies the associated notion of frequency response determined below. Equation (9) also shows how to perform a convolution involving Dirac delta functions on $\mathbb{R}_{S}^{+}, \mathbb{R}_{A}^{+}$. Namely, for $a>0$ :

$$
\begin{aligned}
\delta(t-a) *_{S / A} x(t) & =\frac{1}{2}\left(x_{S / A}(t-a)+x_{S / A}(t+a)\right), \\
h(t) *_{S / A} \delta(t-a) & =\frac{1}{2}\left(h_{S}(t-a) \pm h_{S}(t+a)\right)
\end{aligned}
$$


TABLE III

ESSENTIAL CONCEPTS FOR THE INFINITE CONTINUOUS SPACE MODELS. S DENOTES A SYMMETRIC AND A AN ANTISYMMETRIC EXTENSION.

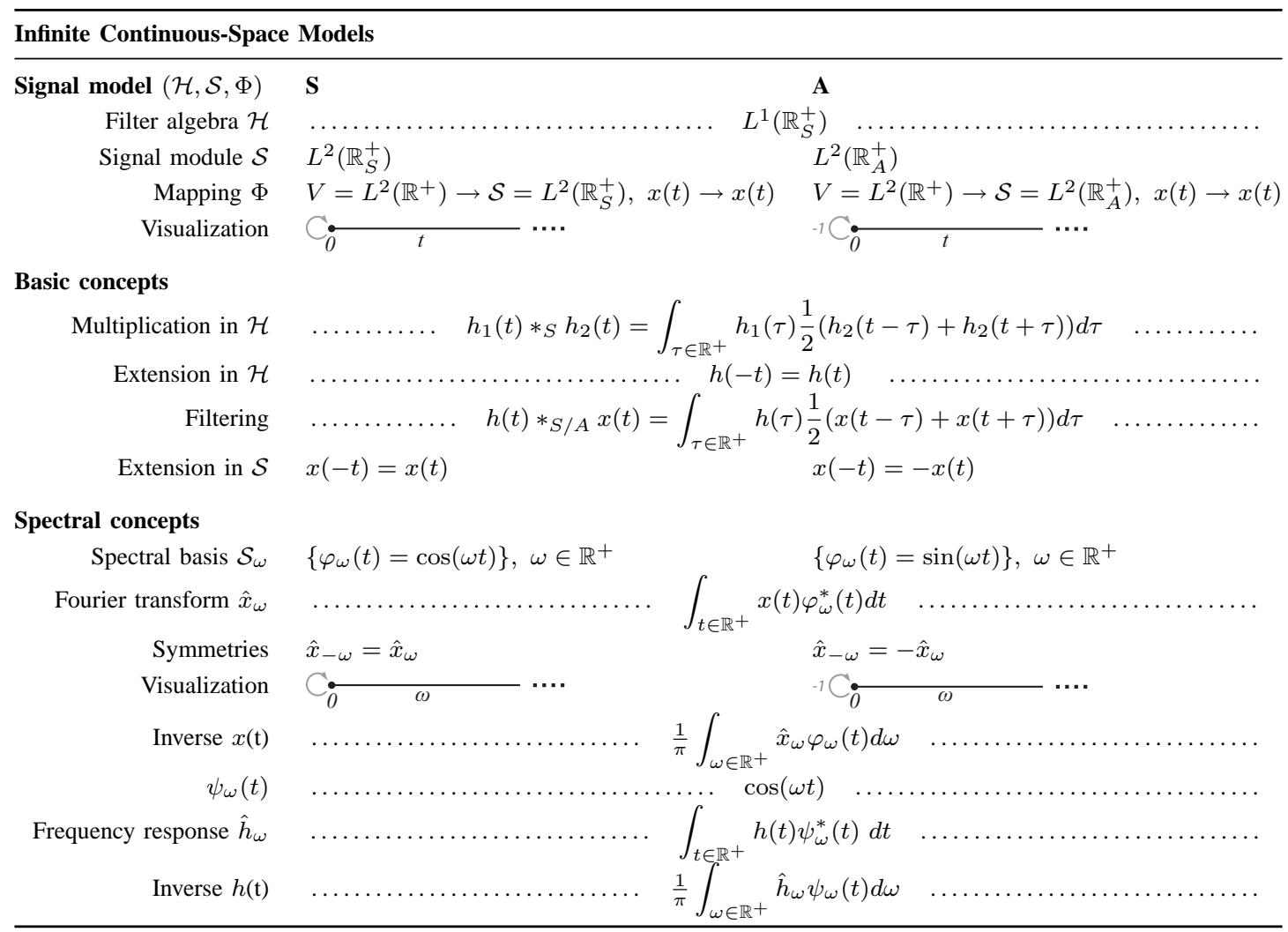

and for $a=0$ (note that $\delta(t)$ is not in $\mathbb{R}_{A}^{+}$):

$$
\delta(t) *_{S / A} x(t)=\frac{1}{2} x(t), \quad h(t) *_{S} \delta(t)=\frac{1}{2} h(t) .
$$

In summary, we get the two space models shown in Table III. Note that they share the same filter algebra (symmetric filters) but have different signal modules (symmetric and antisymmetric signals). Also note that the notions of convolution are well-defined since the convolution of symmetric functions is again symmetric and the convolution of a symmetric and an antisymmetric function is again antisymmetric.

We now proceed by deriving all the other concepts in the table, and immediately see how the space models differ from the time models (compare Table I with Table III). For example, the invariant subspaces under filtering are spanned by cosines for the $\mathrm{S}$ model as shown by $(a>0)$

$$
\delta(t-a) *_{S} \cos (\omega t)=\cos (a t) \cos (\omega t)
$$

and by sines for the A model. The associated Fourier transforms hence take two different forms we call space Fourier transform $S$ (SFT-S) and space Fourier transform A (SFT-A).

In contrast, the frequency response for both models, found by computing the eigenvalues of the respective Fourier basis under filtering as in (12), takes the same form in both cases and is computed exactly as the SFT-S.

Note that the SFT-S and SFT-A are equal to what is called the Fourier cosine transform and the Fourier sine transform in [3]. Hence, as an aside, Table III establishes them as Fourier transforms for properly chosen signal models, and identifies the associated notions of shift, convolution and others.

\section{SAmpling Infinite Continuous Models}

We have already commented on the fact that the three discrete and finite variants of time signal processing in Fig. 1 can be derived from the infinite continuous-time model (Table I) through sampling time, frequency, or both. To do the same for the space models in Table III, we first need a sampling procedure that is general enough to be applicable to other, nontime, signal models including space. We first establish this procedure using infinite continuous-time case as example. Along the way, we will point out subtleties, which, although obvious in time, might not feel that natural in space, and thus, the whole discussion in time is of educational value. The formulation of the sampling theorem most often encountered is due to Shannon [20]; the result is also attributed (by Shannon himself) to Whittaker [21], Kotelnikov [22] and Nyquist [23]. The standard derivation of the sampling theorem can be found in numerous texts ([24], [25], for example).

\section{A. Sampling Infinite Continuous Time}

We derive the sampling theorem for the infinite continuoustime model (Table I) in steps and summarize the results in Table IV. We do it in a slightly nonstandard way; this is done so that the steps can be easily replicated in other signal models.

Select the shift. We choose a shift among those in (1), which means fixing $T$.

Sample the signal and compute Fourier transform. We select a set of locations $t_{n} \in \mathbb{R}$ and require that it be closed under the selected shift by $T$. Hence, the $t_{n}$ have to 
TABLE IV

ESSENTIAL CONCEPTS FOR SAMPLING INFINITE CONTINUOUS TIME.

\begin{tabular}{|c|c|}
\hline Sampling Infinite Continuous & Time \\
\hline \multicolumn{2}{|l|}{ Sampling concepts } \\
\hline Sampling period & $T$ \\
\hline Sampling points $t_{n}, n \in \mathbb{Z}$ & $n T$ \\
\hline Sampled signal $x_{T}(t)$ & $\sum_{n \in \mathbb{Z}} x\left(t_{n}\right) \delta\left(t-t_{n}\right)$ \\
\hline Visualization & $\cdots \cdot \longrightarrow \cdot \overrightarrow{n T}(n+\vec{l}+\vec{\longrightarrow} \cdot \cdots \cdot$ \\
\hline \multicolumn{2}{|l|}{ Spectral concepts } \\
\hline$\varphi_{\omega, n}$ & $e^{j \omega t_{n}}$ \\
\hline Fourier transform $\hat{x}_{T, \omega}$ & $\sum_{n \in \mathbb{Z}} x\left(t_{n}\right) \varphi_{\omega, n}^{*}$ \\
\hline Symmetries & $\hat{x}_{T, m \frac{2 \pi}{T}+\omega}=\hat{x}_{T, \omega}$ \\
\hline Visualization & $\stackrel{\overrightarrow{0}}{\longrightarrow} \underset{2 \pi / T}{\longrightarrow}$ \\
\hline \multicolumn{2}{|l|}{ Sampling theorem concepts } \\
\hline Nyquist band $\Omega_{\mathrm{Nyq}}$ & $\left\{\omega|0 \leq| \omega \mid \leq \frac{\pi}{T}\right\}$ \\
\hline $\begin{array}{r}\text { Sinc filter } \hat{l}_{\omega} \\
l(t)\end{array}$ & $\begin{array}{l} \begin{cases}T & \omega \in \Omega_{\mathrm{Nyq}} \\
0 & \text { otherwise. }\end{cases} \\
\frac{\sin \left(\frac{\pi}{T} t\right)}{\frac{\pi}{T} t}\end{array}$ \\
\hline $\begin{array}{r}\text { Bandlimited subspace } \mathcal{S}_{B L} \\
\text { Basis } b \text { for } \mathcal{S}_{B L}\end{array}$ & $\begin{array}{l}\left\{x \in \mathcal{S} \mid \hat{x}_{\omega}=0, \omega \notin \Omega_{\mathrm{Nyq}}\right\} \\
\left\{l\left(t-t_{n}\right) \mid n \in \mathbb{Z}\right\}\end{array}$ \\
\hline \multicolumn{2}{|c|}{ Sampling theorem For $x(t) \in \mathcal{S}_{B L}: \quad x(t)=\sum_{n \in \mathbb{Z}} x\left(t_{n}\right) l\left(t-t_{n}\right)$} \\
\hline
\end{tabular}

be equidistant. The relative placement with respect to 0 is irrelevant, and thus, we assume that the signal is sampled at integer multiples of $T, t_{n}=n T, n \in \mathbb{Z}$. Sampling can then be described as follows, resulting in the sampled signal $x_{T}(t)$ :

$$
x_{T}(t)=\sum_{n \in \mathbb{Z}} x\left(t_{n}\right) \delta\left(t-t_{n}\right) .
$$

Usually, the sampling process is described as multiplication of the signal by a train of Dirac delta functions, $x_{T}(t)=$ $x(t)\left(\sum_{n \in \mathbb{Z}} \delta\left(t-t_{n}\right)\right)$, and then the Poisson summation formula is used to obtain the sampling theorem. Since the multiplication of signals is strictly speaking outside the signalmodel framework and since we do not want to derive a Poisson formula for every signal model, instead, we compute the FT of the sampled signal directly:

$$
\begin{aligned}
\hat{x}_{T, \omega} & =\int_{t \in \mathbb{R}} x_{T}(t) e^{-j \omega t} d t \\
& =\int_{t \in \mathbb{R}} \sum_{n \in \mathbb{Z}} x\left(t_{n}\right) \delta\left(t-t_{n}\right) e^{-j \omega t} d t \\
& =\sum_{n \in \mathbb{Z}} x\left(t_{n}\right) e^{-j \omega n T},
\end{aligned}
$$

In the above, we used that $\int_{t \in \mathbb{R}} \delta\left(t-T_{0}\right) x(t) d t=x\left(T_{0}\right)$, and $t_{n}=n T$. Setting $x_{n}=x\left(t_{n}\right)$, we recognize the above as the discrete-time Fourier transform (DTFT) of the sequence $x\left(t_{n}\right)$ in $\ell^{2}(\mathbb{Z})$.

Find the symmetries in the Fourier transform. The spectrum of the sampled signal contains redundancy that manifests itself as symmetries. The purpose of this step is to find these symmetries and with it the largest non-redundant interval that these symmetries extend to the entire domain $($ here $\mathbb{R})$. The signal can then be reconstructed from this interval that we will call Nyquist band.

Inspecting (14) shows that

$$
\hat{x}_{T, m \frac{2 \pi}{T}+\omega}=\hat{x}_{T, \omega},
$$

$m \in \mathbb{Z}$, which means that the resulting Fourier transform is $(2 \pi / T)$-periodic. As a consequence, any interval of length $2 \pi / T$ could be chosen as Nyquist band. The usual choice [24] is the symmetric

$$
\Omega_{\mathrm{Nyq}}=\left\{\omega|0 \leq| \omega \mid \leq \frac{\pi}{T}\right\} .
$$

and $\pi / T$ is then called the Nyquist frequency. We will not use this term in the remaining paper since the spectra of the space models considered later are right-sided only: hence, intervals symmetric around zero cannot be chosen since negative frequencies do not exist. To solve this problem, we will work with the notion of the Nyquist band, which is unambiguous.

In Table IV, $[0,2 \pi / T]$ is shown as Nyquist band; the backwards arrow shows the periodic extension to $\mathbb{R}$. For the space models considered later, other, non-periodic symmetries will occur.

Extract the original spectrum. Assuming that the signal is bandlimited to the Nyquist band, for reconstruction, we need to extract only that band by applying an ideal lowpass filter supported in the Nyquist band. In the time domain, this is equivalent to filtering the signal by the inverse frequency response $^{6}$ of this lowpass filter. This is the sinc filter $l(t)$ we give in (16); it is indeed exactly 1 at $t_{0}=0$ and 0 at all other sampling points $t_{n}=n T$, or, $l\left(t_{n}\right)=\delta_{n}$, as given in Table IV. The sinc filter and its frequency response are:

$$
\hat{l}_{\omega}=\left\{\begin{array}{ll}
T & \omega \in \Omega_{\mathrm{Nyq}}, \\
0 & \text { otherwise, }
\end{array} \quad l(t)=\frac{\sin \left(\frac{\pi}{T} t\right)}{\frac{\pi}{T} t} .\right.
$$

Sampling theorem. The above extraction process is equivalent to filtering the sampled signal with $l(t)$ :

$$
\begin{aligned}
x(t) & =l(t) * x_{T}(t)=\int_{\tau \in \mathbb{R}} l(\tau) x_{T}(t-\tau) d \tau \\
& =\int_{\tau \in \mathbb{R}} l(\tau) \sum_{n \in \mathbb{Z}} x\left(t_{n}\right) \delta\left(t-\tau-t_{n}\right) d \tau
\end{aligned}
$$

which, after exchanging integral and sum, leads to the expression for the sampling theorem: ${ }^{7}$

Theorem 1 (Sampling theorem: ICT Model) For a signal $x(t)$ bandlimited to the Nyquist band:

$$
x(t)=\sum_{n \in \mathbb{Z}} x\left(t_{n}\right) l\left(t-t_{n}\right) .
$$

Theorem 1 shows that the space $\mathcal{S}_{B L}=\{x \in \mathcal{S} \mid$ $\left.\hat{x}_{\omega}=0, \omega \notin \Omega_{\mathrm{Nyq}}\right\}$ of bandlimited signals is spanned by the

${ }^{6}$ Note that here, we are making the distinction between Fourier transform, applied to signals, and frequency response, applied to filters. In continuous time, these two are identical; however, this will not be the case for all the signal models we are considering, as we will see later.

${ }^{7}$ We will denote by I: infinite, F: finite, C: continuous, T: time, S: space. 

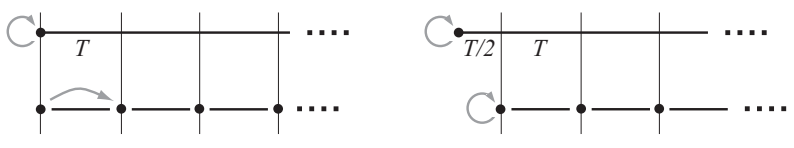

Fig. 3. Two ways (W on the left and $\mathrm{H}$ on the right) of sampling the infinite space model S. Left: Start at $t_{0}=0$ and sample $T$ apart. Right: Start at $t_{0}=T / 2$ and sample $T$ apart.

orthogonal set $l(t) * \delta\left(t_{n}\right)=l\left(t-t_{n}\right), n \in \mathbb{Z}$. Note that these $l\left(t-t_{n}\right)$, as used in (18), are signals now and not filters.

Comment. Sampling infinite time yields the infinite discrete model and the finite continuous model as its spectral domain. This motivates the introduction of the latter, but, more importantly, it is needed to obtain the finite discrete model (and with it the DFT) again by sampling.

By sampling the infinite continuous-space model, we thus expect to not only find infinite discrete-space models, but also the proper finite continuous models that need to be sampled to obtain the sixteen DCTs/DSTs as the Fourier transforms for the corresponding finite discrete-space models.

\section{B. Sampling Infinite Continuous Space}

We now repeat the above process for the two infinite space models defined in Table III. We cover S in detail and only briefly point out differences in A. The results are summarized in Tables V and VI.

In the infinite space model $\mathrm{S}$, both the signals and the filters are defined on $\mathbb{R}_{S}^{+}$visualized in Table III.

Select the shift. We select a space shift among those in (3), which means fixing $T$.

Sample the signal and compute Fourier transform. We select a set of sampling locations $t_{n}$ closed under the space shift by $T$, required to be equidistant, just as for the time model. It turns out there are only two possible choices, depicted in Fig. 3, namely starting at 0 or $T / 2$. For every other choice, the leftmost point will not have the distance of a multiple of $T$ to itself (walking left and being reflected due to the boundary condition). We refer to the two choices as whole-point sampling $(W)$ and half-point sampling $(H)$ following [11]. In the former case, samples are taken at $t_{n}=n T, n \geq 0$; in the the latter case, samples are taken at $t_{n}=n T+T / 2, n \geq 0$.

We proceed by considering the $\mathrm{W}$ case in detail; $\mathrm{H}$ is similar and also given in Table $\mathrm{V}$.

We sample the signal $x(t)$ at $t_{n}=n T, n \geq 0$ and get

$$
x_{T}(t)=\sum_{n=0}^{\infty} x\left(t_{n}\right) \delta\left(t-t_{n}\right)
$$

As in the time case, we proceed by directly computing the Fourier transform of $x_{T}(t)$. The Fourier transform to be used is the one associated with the space model $\mathrm{S}$ from Table III:

$$
\begin{aligned}
\hat{x}_{T, \omega} & =\int_{t \in \mathbb{R}^{+}} x_{T}(t) \cos (\omega t) d t \\
& =\int_{t \in \mathbb{R}^{+}}\left(\sum_{n=0}^{\infty} x\left(t_{n}\right) \delta\left(t-t_{n}\right)\right) \cos (\omega t) d t \\
& =\frac{1}{2} x\left(t_{0}\right)+\sum_{n=1}^{\infty} x\left(t_{n}\right) \cos \left(\omega t_{n}\right) .
\end{aligned}
$$

The factor $1 / 2$ associated with the first sample appears for the same reasons as in (11). We could call the above a discretespace Fourier transform of the sequence $x\left(t_{n}\right), n \geq 0$.

Find the symmetries in the Fourier transform. We now need to find the Nyquist band of the sampled signal $\hat{x}_{T, \omega}$, i.e., the largest interval on which the spectrum is nonredundant. As before, this is done by finding the symmetries.

Inspecting (20) reveals the symmetry

$$
\hat{x}_{T, 2 m \frac{2 \pi}{T} \pm \omega}=\hat{x}_{T, \omega}, \quad m \in \mathbb{Z} .
$$

Hence, we can visualize the spectrum as living on the interval $[0, \pi / T)$, extended symmetrically to the left and to the right. In Table V, this domain is visualized as a line between $[0, \pi / T)$ with loops on the left and right with weights 1 . The Nyquist band is accordingly $\Omega_{\mathrm{Nyq}}=\left\{\omega \mid 0 \leq \omega \leq \frac{\pi}{T}\right\}$.

Extract the original spectrum. We extract the Nyquist band by applying an ideal lowpass filter with frequency response $\hat{l}_{\omega}$ supported only in the Nyquist band. Applying the inverse frequency response from Table III yields the same result as in the time case (16), only the filter is viewed as a function on $\mathbb{R}_{S}^{+}$:

$$
\hat{l}_{\omega}=\left\{\begin{array}{ll}
T & \omega \in \Omega_{\mathrm{Nyq}}, \\
0 & \text { otherwise, }
\end{array} \quad l(t)=\frac{\sin \left(\frac{\pi}{T} t\right)}{\frac{\pi}{T} t} .\right.
$$

As in time, the interpolating sinc is indeed 1 at $t_{0}=0$ and 0 at all other sampling points $t_{n}=n T$.

Sampling theorem. The extraction process which results in the original signal, is pointwise multiplication of the sampled signal $\hat{x}_{T, \omega}$ with the sinc filter $\hat{l}_{\omega}$ in the Fourier domain, or, equivalently, $\mathrm{S}$ convolution in the original domain:

$$
\begin{aligned}
x(t) & =l(t) *_{S} x_{T}(t)=l(t) *_{S} \sum_{n=0}^{\infty} x\left(t_{n}\right) \delta\left(t-t_{n}\right) \\
& =\sum_{n=0}^{\infty} x\left(t_{n}\right)\left(l(t) *_{S} \delta\left(t-t_{n}\right)\right),
\end{aligned}
$$

where we have used linearity of the convolution. Using (10) and (11) we get the sampling theorem:

Theorem 2 (Sampling theorem: ICS-S Model with $W$ ) For a signal $x(t)$ bandlimited to the Nyquist band:

$$
x(t)=\frac{1}{2} x\left(t_{0}\right) l(t)+\sum_{n=1}^{\infty} x\left(t_{n}\right) \frac{1}{2}\left(l\left(t-t_{n}\right)+l\left(t+t_{n}\right)\right) .
$$

Theorem 2 shows that the set of signals bandlimited to the Nyquist band is spanned by the $l(t) *_{S} \delta\left(t-t_{n}\right)$, which are space-shifted copies of the sinc filter in (21). As in time, these are signals (not filters) and orthogonal. 
TABLE V

ESSENTIAL CONCEPTS FOR SAMPLING INFINITE CONTINUOUS SPACE WITH EXTENSION S.

Sampling Infinite Continuous Space: Model S (Symmetric Extension)

W H

Sampling concepts

Sampling period $T$

Sampling points $t_{n}, n \geq 0 \quad n T$

Sampled signal $x_{T}(t)$

Visualization
$\mathbf{H}$

$T$

$n T+\frac{T}{2}$

$\sum_{n=0}^{\infty} x\left(t_{n}\right) \delta\left(t-t_{n}\right)$

$\mathrm{C}_{T / 2} \cdot \overline{3 T / 2} \cdot \overline{5 T / 2} \cdot \cdots$

Spectral concepts

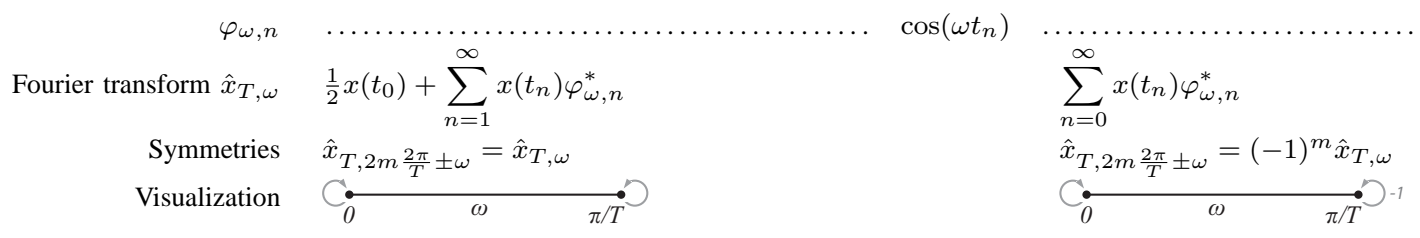

Sampling theorem concepts

Nyquist band $\Omega_{\mathrm{Nyq}}$

Sinc filter $\hat{l}_{\omega}$

$l(t)$

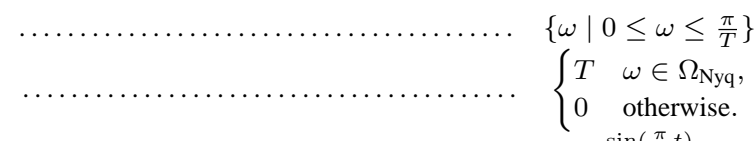

$\left\{\omega \mid 0 \leq \omega \leq \frac{\pi}{T}\right\}$

(0) otherwise.

Bandlimited subspace $\mathcal{S}_{B L}$

$\ldots \ldots \ldots \ldots \ldots \ldots \ldots \ldots \ldots \ldots \ldots \ldots \ldots \ldots \ldots, \quad\left\{x \in \mathcal{S} \mid \hat{x}_{\omega}=0, \omega \notin \Omega_{\mathrm{Nyq}}\right\}$

Basis $b$ for $\mathcal{S}_{B L}$

$\left\{\frac{1}{2} l(t)\right\} \cup\left\{\frac{1}{2}\left(l\left(t-t_{n}\right)+l\left(t+t_{n}\right)\right) \mid n=1, \ldots\right\}$

$\left\{\frac{1}{2}\left(l\left(t-t_{n}\right)+l\left(t+t_{n}\right)\right) \mid n=0, \ldots\right\}$

Sampling theorem For $x(t) \in \mathcal{S}_{B L}: \quad x(t)=\frac{1}{2} x\left(t_{0}\right) l(t)+\sum_{n=1}^{\infty} x\left(t_{n}\right) \frac{1}{2}\left(l\left(t-t_{n}\right)+l\left(t+t_{n}\right)\right) \quad x(t)=\sum_{n=0}^{\infty} x\left(t_{n}\right) \frac{1}{2}\left(l\left(t-t_{n}\right)+l\left(t+t_{n}\right)\right)$

TABLE VI

ESSENTIAL CONCEPTS FOR SAMPLING INFINITE CONTINUOUS SPACE WITH EXTENSION A.

Sampling Infinite Continuous Space Model A (Antisymmetric Extension)

W

Sampling concepts

Sampling period

Sampling points $t_{n}, n \geq 0$

Sampled signal $x_{T}(t)$

Visualization

Spectral concepts

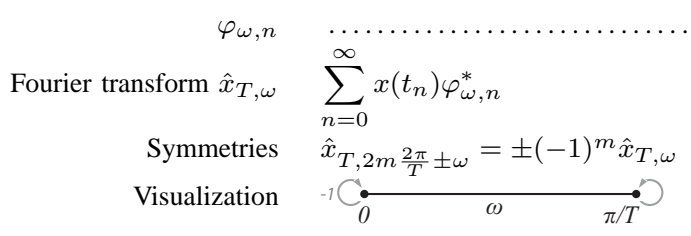

$n T+T \quad n T+\frac{T}{2}$

$\ldots \ldots \ldots \ldots \ldots \ldots \ldots \ldots \sum_{n=0}^{\infty} x\left(t_{n}\right) \delta\left(t-t_{n}\right)$

$\stackrel{\bullet}{T}-\cdot-\cdots \cdot$

Sampling theorem concepts

Nyquist band $\Omega_{\mathrm{Nyq}}$

Sinc filter $\hat{l}_{\omega}$

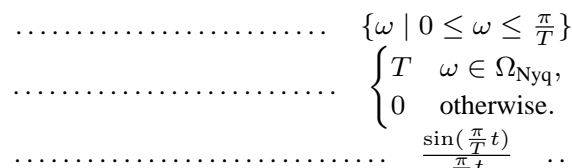

Bandlimited subspace $\mathcal{S}_{B L}$

Basis $b$ for $\mathcal{S}_{B L}$

$\ldots \ldots \ldots \ldots \ldots \ldots . . \quad\left\{x \in \mathcal{S} \mid \hat{x}_{\omega}=0, \omega \notin \Omega_{\mathrm{Nyq}}\right\}$

$\left.\ldots \ldots \ldots \ldots \ldots\} \quad\left\{\frac{1}{2}\left(l\left(t-t_{n}\right)-l\left(t+t_{n}\right)\right)\right) \mid n=0, \ldots\right\}$

Sampling theorem For $x(t) \in \mathcal{S}_{B L}: \quad x(t)=\sum_{n=0}^{\infty} x\left(t_{n}\right) \frac{1}{2}\left(l\left(t-t_{n}\right)-l\left(t+t_{n}\right)\right)$ 

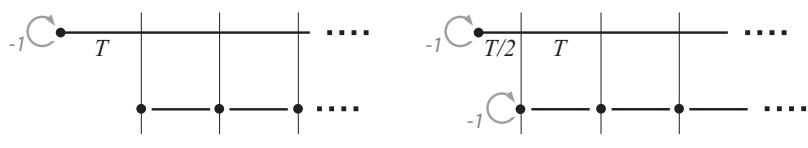

Fig. 4. Two ways (W on the left and $\mathrm{H}$ on the right) of sampling the infinite space model A. Left: Start at $t_{0}=T$ and sample $T$ apart. Right: Start at $t_{0}=T / 2$ and sample $T$ apart.

Half-point sampling. Half-point sampling starts sampling at $t=T / 2$, which is at distance $T$ from its mirror image $-T / 2$. This choice changes the associated discrete-space Fourier transform and also the symmetry in the spectrum of the sampled signal:

$$
\hat{x}_{T, 2 m \frac{2 \pi}{T} \pm \omega}=(-1)^{m} \hat{x}_{T, \omega}, \quad m \in \mathbb{Z} .
$$

We depict the spectral domain as a line 0 to $\pi / T$ where the loop on the right side has weight -1 for the antisymmetric extension (Table V, column $\mathrm{H}$ ).

The Nyquist band is exactly the same as for the $\mathrm{W}$ case and hence the sinc filter $l(t)$ is also identical to the $\mathrm{W}$ case. Thus, the sampling theorem takes the following form:

Theorem 3 (Sampling theorem: ICS-S Model with H) For a signal $x(t)$ bandlimited to the Nyquist band:

$$
x(t)=\sum_{n=0}^{\infty} x\left(t_{n}\right) \frac{1}{2}\left(l\left(t-t_{n}\right)+l\left(t+t_{n}\right)\right) .
$$

The space of bandlimited signals is again spanned by $l(t) *_{S}$ $\delta\left(t_{n}\right)$, that is, signals obtained from the template sinc, now space-shifted by the half integers $t_{n}$.

Sampling the infinite space model A. We now briefly discuss sampling the other infinite space model, that with the antisymmetric extension from Table III. As we noted before, first big differences occur here: filter ans signal space are different and the Fourier transform of a signal is computed differently than the frequency response of a filter. Note that all signals are 0 at $t=0$, which can thus be omitted as a sampling point. The two equidistant choices are shown in Fig. 4.

The sampling process is summarized in Table VI; as the steps are identical to what we have just seen, we only briefly point out the differences. First, we get two additional versions of a discrete-space Fourier transform and two new forms of symmetry in the frequency domain. However, the Nyquist band is the same as before, and since the filter spaces for $\mathrm{S}$ and $\mathrm{A}$ are the same (Table III), the sinc filter is the same as before. However, the space of bandlimited signals now takes a different form: it is again spanned by signals obtained from the template sinc filter convolved with all $\delta\left(t-t_{n}\right)$ which, using (10), evaluates to

$$
l(t) *_{A} \delta\left(t-t_{n}\right)=\frac{1}{2}\left(l\left(t-t_{n}\right)-l\left(t+t_{n}\right)\right) .
$$

\section{Finite Continuous-Space Models}

Sampling the the infinite continuous-space models in the previous section yielded four infinite discrete-space models and their corresponding finite continuous spectra visualized in Tables V and VI. Since our goal is to sample those (obtaining the sixteen DCTs/DSTs as one outcome) we first formally define the four finite continuous-space models. They are the space equivalent of the finite continuous-time model in Table II.

The four models are summarized in Table VII. Note that the visualizations of the signal models correspond to the visualizations of the spectra in Tables V and VI. The signal domains are intervals that on each side are either symmetrically or antisymmetrically extended yielding the four choices denoted with SS, SA, AS, AA. The filter algebra is the same in all cases. Note that all models are well-defined since spaceshifting (3) an SS/SA/AS/AA signal yields a signal with the same symmetry.

We cover only the SS model in detail; we briefly touch upon others.

\section{A. Finite Space Model SS}

Beside the inherent "beauty" of both extensions being symmetric, we have a practical reason for dealing with this case in detail. As we will see later, one of its sampled models leads to the well-known DCT (of type 2), which has been used in image processing for a long time.

Signal model and basic concepts. Signal and filter space are $L^{1}\left(\mathbb{I}_{S S}\right)$, which consist of functions on the interval $\mathbb{I}=$ $[0, I]$ symmetrically extended in both directions. Formally, a signal $x(t)$ on $\mathbb{I}$ is $\mathrm{SS}$ extended as

$$
x_{S S}(2 k I \pm t)=x(t), \quad k \in \mathbb{Z} .
$$

Note that this is the same as first $\mathrm{S}$-extending $x(t)$ to $[-I, I]$ and then periodically extending the result: $\left(x_{S}\right)_{P}=x_{S S}$, that is, SS signals have the period $2 I$. Filters for the model have the same extension. SS convolution, defined in Table VII, can be related to ordinary convolution and $\mathrm{S}$ convolution as follows:

$$
h *_{S S} x=\frac{1}{2}\left(h_{S} * x_{S S}\right)=h *_{S} x_{S S},
$$

where in the last term, $x_{S S}$ is viewed as a function on $\mathbb{R}^{+}$ and in all cases the result is viewed as a function on $\mathbb{I}$.

As in Section III, we have to pay particular attention to convolving with Dirac delta functions; the results are derived using (24). For $a \in(0, I)$, as expected,

$$
\begin{aligned}
\delta(t-a) *_{S S} x(t) & =\frac{1}{2}\left(x_{S S}(t-a)+x_{S S}(t+a)\right), \\
h(t) *_{S S} \delta(t-a) & =\frac{1}{2}\left(h_{S S}(t-a)+h_{S S}(t+a)\right) .
\end{aligned}
$$

For $a=0, I$, the result is analogous to (11) and for the same reasons:

$$
\begin{aligned}
& \delta(t) *_{S S} x(t)=\frac{1}{2} x(t), \delta(t-I) *_{S S} x(t)=\frac{1}{2} x(t-I), \\
& h(t) *_{S S} \delta(t)=\frac{1}{2} h(t), h(t) *_{S S} \delta(t-I)=\frac{1}{2} h(t-I) .
\end{aligned}
$$

Spectral concepts. The eigenspaces under the above filtering are

$$
\mathcal{S}_{k}=\left\{\varphi_{k}(t)=\cos \left(\frac{k \pi}{I} t\right)\right\},
$$


TABLE VII

ESSENTIAL CONCEPTS FOR THE FINITE CONTINUOUS SPACE MODELS.

\begin{tabular}{|c|c|c|c|c|}
\hline \multicolumn{5}{|c|}{ Finite Continuous-Space Models } \\
\hline Signal model $(\mathcal{H}, \mathcal{S}, \Phi)$ & SS & SA & $\mathbf{A S}$ & $\mathbf{A A}$ \\
\hline Filter algebra $\mathcal{H}$ & 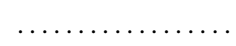 & $\cdots$ & $S S)$ & \\
\hline Signal module $\mathcal{S}$ & $L^{1}\left(\mathbb{I}_{S S}\right)$ & $L^{1}\left(\mathbb{I}_{S A}\right)$ & $L^{1}\left(\mathbb{I}_{A S}\right)$ & $L^{1}\left(\mathbb{I}_{A A}\right)$ \\
\hline Mapping $\Phi$ & $L^{1}(\mathbb{I}) \rightarrow L^{1}\left(\mathbb{I}_{S S}\right)$ & $L^{1}(\mathbb{I}) \rightarrow L^{1}\left(\mathbb{I}_{S A}\right)$ & $L^{1}(\mathbb{I}) \rightarrow L^{1}\left(\mathbb{I}_{A S}\right)$ & $L^{1}(\mathbb{I}) \rightarrow L^{1}\left(\mathbb{I}_{A A}\right)$ \\
\hline Visualization & $C_{0}^{-}$ & $\mathrm{C}_{0}^{-}$ & ${ }^{-1} C_{0}$ & ${ }^{-1} C_{0}$ \\
\hline
\end{tabular}

Basic concepts

Multiplication in $\mathcal{H}$

$$
h_{1}(t) *_{S S} h_{2}(t)=\int_{\tau \in \mathbb{I}} h_{1}(\tau) \frac{1}{2}\left(h_{2}(t-\tau)+h_{2}(t+\tau)\right) d \tau
$$

Extension in $\mathcal{H}$

Filtering

$$
\begin{aligned}
& h(t) *_{S S / S A / A S / A A} x(t)=\int_{\tau \in \mathbb{I}} h(\tau) \frac{1}{2}(x(t-\tau)+x(t+\tau)) d \tau
\end{aligned}
$$

Extension in $\mathcal{S} \quad x(2 k I \pm t)=x(t)$

$x(2 k I \pm t)=(-1)^{k} x(t)$

$x(2 k I \pm t)= \pm(-1)^{k} x(t)$

$x(2 k I \pm t)= \pm x(t)$

\section{Spectral concepts}

Spectral basis $\mathcal{S}_{k}, k \geq 0$

Fourier transform $\hat{x}_{k}$

Visualization

$\left\{\varphi_{k}(t)=\cos \left(\frac{k \pi}{I} t\right)\right\}$

$\left\{\varphi_{k}(t)=\cos \left(\frac{\left(k+\frac{1}{2}\right) \pi}{I} t\right)\right\}$

$\left\{\varphi_{k}(t)=\sin \left(\frac{\left(k+\frac{1}{2}\right) \pi}{I} t\right)\right\}$

$\left\{\varphi_{k}(t)=\sin \left(\frac{k \pi}{I} t\right)\right\}$

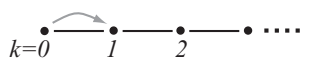

$\int_{t \in \mathbb{I}} x(t) \varphi_{k}^{*}(t) d t$

Inverse $x(\mathrm{t})$

$\frac{1}{I} \hat{x}_{0}+\frac{2}{I} \sum_{k=1}^{\infty} \hat{x}_{k} \varphi_{k}(t)$

Symmetries

$\hat{x}_{-k}=\hat{x}_{k}$

$\psi_{k}(t)$

$\cos \left(\frac{k \pi}{I} t\right)$

Frequency response $\hat{h}_{k}$

Inverse $h(\mathrm{t})$

$\frac{1}{I} \hat{h}_{0}+\frac{2}{I} \sum_{k=1}^{\infty} \hat{h}_{k} \psi_{k}(t)$

${ }_{k=0}-i-i-\cdots$

$\frac{2}{I} \sum_{k=0}^{\infty} \hat{x}_{k} \varphi_{k}(t)$

$\hat{x}_{-k}=\hat{x}_{k-1}$

$\cos \left(\frac{\left(k+\frac{1}{2}\right) \pi}{I} t\right)$

${ }_{k}{ }_{k=0}-{ }_{i}-\cdots$

$\frac{2}{I} \sum_{k=0}^{\infty} \hat{x}_{k} \varphi_{k}(t)$

$\hat{x}_{-k}=-\hat{x}_{k-1}$

$\cos \left(\frac{\left(k+\frac{1}{2}\right) \pi}{I} t\right)$

$\int_{t \in \mathbb{I}} h(t) \psi_{k}^{*}(t) d t$

$\frac{2}{I} \sum_{k=0}^{\infty} \hat{h}_{k} \psi_{k}(t)$

$\frac{2}{I} \sum_{k=0}^{\infty} \hat{h}_{k} \psi_{k}(t)$ $k=\dot{0}-\stackrel{i}{i} \cdots \cdot$

$\frac{2}{I} \sum_{k=1}^{\infty} \hat{x}_{k} \varphi_{k}(t)$

$\hat{x}_{-k}=-\hat{x}_{k}$

$\cos \left(\frac{k \pi}{I} t\right)$

$k \in \mathbb{Z}$. Let $x(t)=\varphi_{k}(t) \in \mathcal{S}_{k}$ and $h(t)$ any filter, then

Convolution can be related to ordinary convolution as

$$
h(t) *_{S S} \varphi_{k}(t)=\frac{1}{I} \int_{\tau \in \mathbb{I}} h(\tau) \frac{1}{2}\left(\cos \left(\frac{k \pi}{I}(t-\tau)\right)+\cos \left(\frac{k \pi}{I}(t+\tau)\right)\right) d h *_{S S / S A / A S / A A} x=\frac{1}{2}\left(h_{S} * x_{S S / S A / A S / A A}\right) .
$$

$=\frac{1}{I} \int_{\tau \in \mathbb{I}} h(\tau) \cos \left(\frac{k \pi}{I} \tau\right) \cos \left(\frac{k \pi}{I} t\right) d \tau$

$=\varphi_{k}(t) c \int_{\tau \in \mathbb{I}} h(\tau) \cos \left(\frac{k \pi}{I} \tau\right) d \tau$

$=\varphi_{k}(t) \underbrace{\left.c \int_{\tau \in \mathbb{I}} h(\tau) \varphi_{k}^{*}(\tau)\right) d \tau}_{\hat{h}_{k}}=\hat{h}_{k} \varphi_{k}(t)$.

This conforms with the well-known fact that periodic functions that are also symmetric (here $x_{S S}=\left(x_{S}\right)_{P}$ ) have a Fourier series consisting of cosines only. The derivation also yields the frequency response $\hat{h}_{k}$, computed the same way as the Fourier transform. This is not the case for the SA/AS/AA models.

Note that in (27) $\mathcal{S}_{-1}=\mathcal{S}_{1}$ is whole-point symmetric; thus the structure of the spectrum is as shown in Table VII. This is expected since it corresponds to the $\mathrm{W}$ column of Table $\mathrm{V}$.

\section{B. Other Models}

We briefly discuss the other models in Table VII.

Signal model and basic concepts. First, we note that due to the antisymmetric extension, the AS model requires $x(0)=0$, the SA model $x(I)=0$, and the AA model both. The AA model has period $2 I$ and the SA and AS model the period $4 I$.
This equation can be used to compute the convolution with Dirac delta functions. For example, for $a \in(0, I)$,

$$
\begin{aligned}
& h *_{S S / A A} \delta(t-a)=\frac{1}{2}\left(h_{S S}(t-a) \pm h_{S S}(t+a)\right), \\
& h *_{S A / A S} \delta(t-a)=\frac{1}{2}\left(h_{S A}(t-a) \pm h_{S A}(t+a)\right),
\end{aligned}
$$

and for $a=0, I$,

$h(t) *_{S A} \delta(t)=\frac{1}{2} h(t), \quad h(t) *_{A S} \delta(t-I)=-\frac{1}{2} h(t-I)$.

Spectral concepts. The subspaces invariant under filtering take different forms for the four models as shown in Table VII. However, the frequency response takes only two different forms: it is the same for SS and AA and for SA and AS. Except for SS and SA, it is computed differently than the Fourier transform.

Interestingly, the inverse frequency response for the SA and AS model will yield an SA function (since all $\psi_{k}$ have this property in this case), which hence satisfies $h(I)=0$. Hence it will reconstruct $h(t)$ only on $[0, I)$ if the sum converges.

\section{Sampling Finite Continuous Models}

In this section we sample finite continuous models following the same steps as before. For illustration, we start with the 
TABLE VIII

ESSENTIAL CONCEPTS FOR SAMPLING FINITE CONTINUOUS TIME.

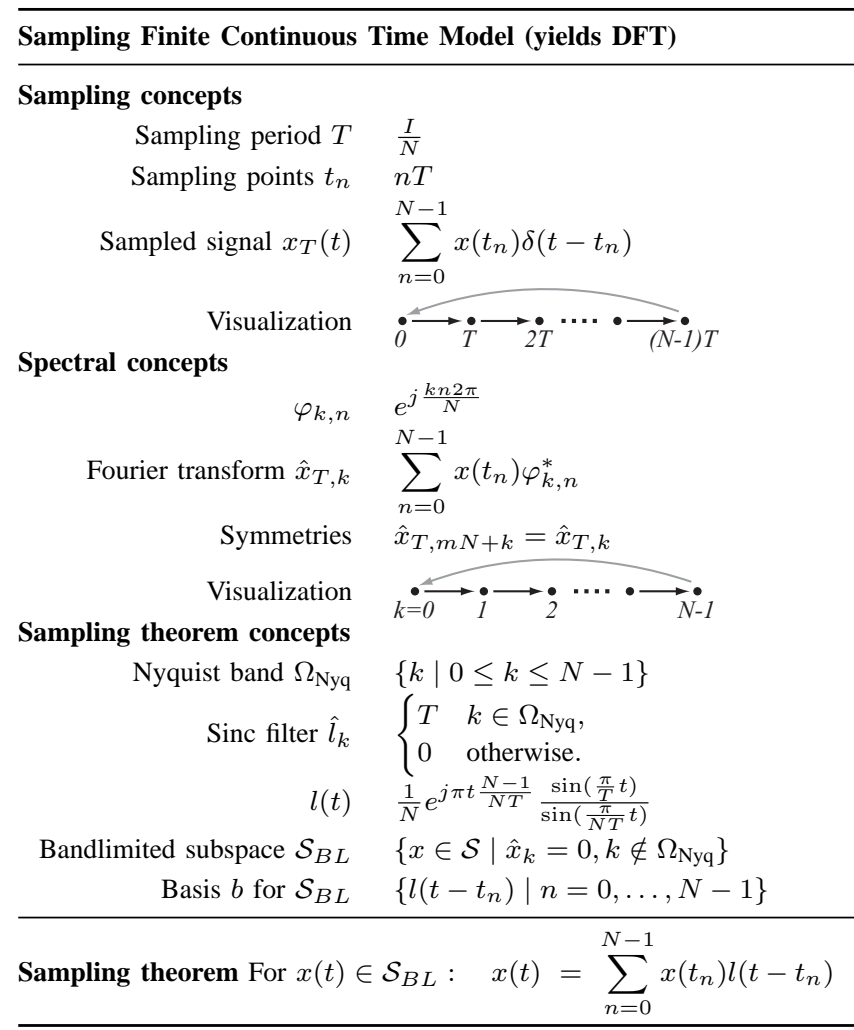

familiar finite time model in Table II and obtain, besides the sampling theorem, the DFT. The same procedure applied to the four finite space models in Table VII will later yield the sixteen DCTs/DSTs.

\section{A. Sampling Finite Continuous Time}

The main concepts we discuss here are summarized in Table VIII.

Select the shift. We select a time shift on the circle, which means fixing $T$.

Sample the signal and compute Fourier transform. The sampling points $t_{n}$ have to be closed under the shift by $T$, hence they have to be equidistant on the circle of length $I$. We start at $0,{ }^{8}$ and choose $N$ samples, that is, $T=I / N$. Sampling yields

$$
x_{T}(t)=\sum_{n=0}^{N-1} x\left(t_{n}\right) \delta\left(t-t_{n}\right)
$$

and the Fourier transform of $x_{T}(t)$ (defined in Table II) is

$$
\hat{x}_{T, k}=\int_{t \in \mathbb{I}} x_{T}(t) e^{-j \frac{2 \pi k}{I} t} d t=\sum_{n=0}^{N-1} x\left(t_{n}\right) e^{-j \frac{k n 2 \pi}{N}} .
$$

Setting $W_{N}=e^{j \frac{2 \pi}{N}}$ and $x_{n}=x\left(t_{n}\right)$, this is precisely the DFT of a sequence of length $N$ :

$$
\hat{x}_{T, k}=\sum_{n=0}^{N-1} x_{n} W_{N}^{-k n} .
$$

\footnotetext{
${ }^{8}$ Different starting points lead to slightly different versions of the DFT.
}

The above process yields the structure of the finite discretetime model (bottom right in Fig. 1). The signal lives on a circle with $N$ points (it is discrete periodic with a fundamental period of length $N$ ) and the shift moves the signal by one sample clockwise. The DFT diagonalizes the adjacency matrix of the circle (the cyclic shift).

Find the symmetries in the Fourier transform. From (32), we see that after sampling, the spectrum becomes periodic:

$$
\hat{x}_{T, m N+k}=\hat{x}_{T, k}, \quad m \in \mathbb{Z} .
$$

Again, this means that we can choose any interval of length $N$ as Nyquist band. To be consistent with the infinite continuoustime case (15), the $N$ points should be symmetric around 0 . However, this would yield a non-standard definition of the DFT. We choose the Nyquist band $\Omega_{\mathrm{Nyq}}=\{k \mid 0 \leq$ $k \leq N-1\}$ to obtain the DFT as defined in almost every signal processing book. Interestingly, this is not consistent with (15), and often produces confusion as our inherent notion of what the natural ordering of frequencies should be (low to high) is disturbed; with this definition of the DFT, the highest frequency is in the middle of the spectrum. Remember, however, that this is just the question of ordering, as any interval of length $N$ would do the trick. This is one reason that in this paper we abandon the terms Nyquist rate and Nyquist frequency in favor of Nyquist band. The other is that space models have no negative frequencies; hence, a symmetric band is inherently not possible.

Extract the original spectrum. Again, assuming a signal bandlimited to the Nyquist band, for reconstruction, we use the appropriate sinc filter $l(t)$ :

$$
\hat{l}_{k}=\left\{\begin{array}{ll}
T & k \in \Omega_{\mathrm{Nyq}}, \\
0 & \text { otherwise. }
\end{array} \quad l(t)=\frac{1}{N} e^{j \pi t \frac{N-1}{N T}} \frac{\sin \left(\frac{\pi}{T} t\right)}{\sin \left(\frac{\pi}{N T} t\right)} .\right.
$$

Inspect $l(t)$ : the numerator provides the zeros at all integer multiples of $T$, including $T=0$. The denominator is 0 at $t=0$ and is then nonzero until $t= \pm N T$, that is, its period is $N$ times larger than that of the numerator. Together they give a value of 1 at $t=0$ and zeros at all integer multiples of $T$, as an interpolating sinc should.

Sampling theorem. The above extraction process is pointwise multiplication of the sampled signal $\hat{x}_{T, k}$ with the sinc filter $\hat{l}_{k}$ in the Fourier domain. This is equivalent to the convolution

$$
\begin{aligned}
x(t) & =\left(l(t) *_{P} x_{T}(t)\right)=\frac{1}{I} \int_{\tau \in \mathbb{I}} l(\tau) x_{T}(t-\tau) d \tau, \\
& =\frac{1}{I} \int_{\tau \in \mathbb{I}} l(\tau) \sum_{n=0}^{N-1} x\left(t_{n}\right) \delta\left(t-\tau-t_{n}\right) d \tau
\end{aligned}
$$

which leads to the expression for the sampling theorem:

Theorem 4 (Sampling theorem: FCT Model) For a signal $x(t)$ bandlimited to the Nyquist band:

$$
x(t)=\sum_{n=0}^{N-1} x\left(t_{n}\right) l\left(t-t_{n}\right) .
$$

The space $\mathcal{S}_{B L}$ of those signals bandlimited exactly to the Nyquist band (see Table VIII), that is, the space of signals 
TABLE IX

ESSENTIAL CONCEPTS FOR SAMPLING FINITE CONTINUOUS SPACE MODEL WITH EXTENSION SS.

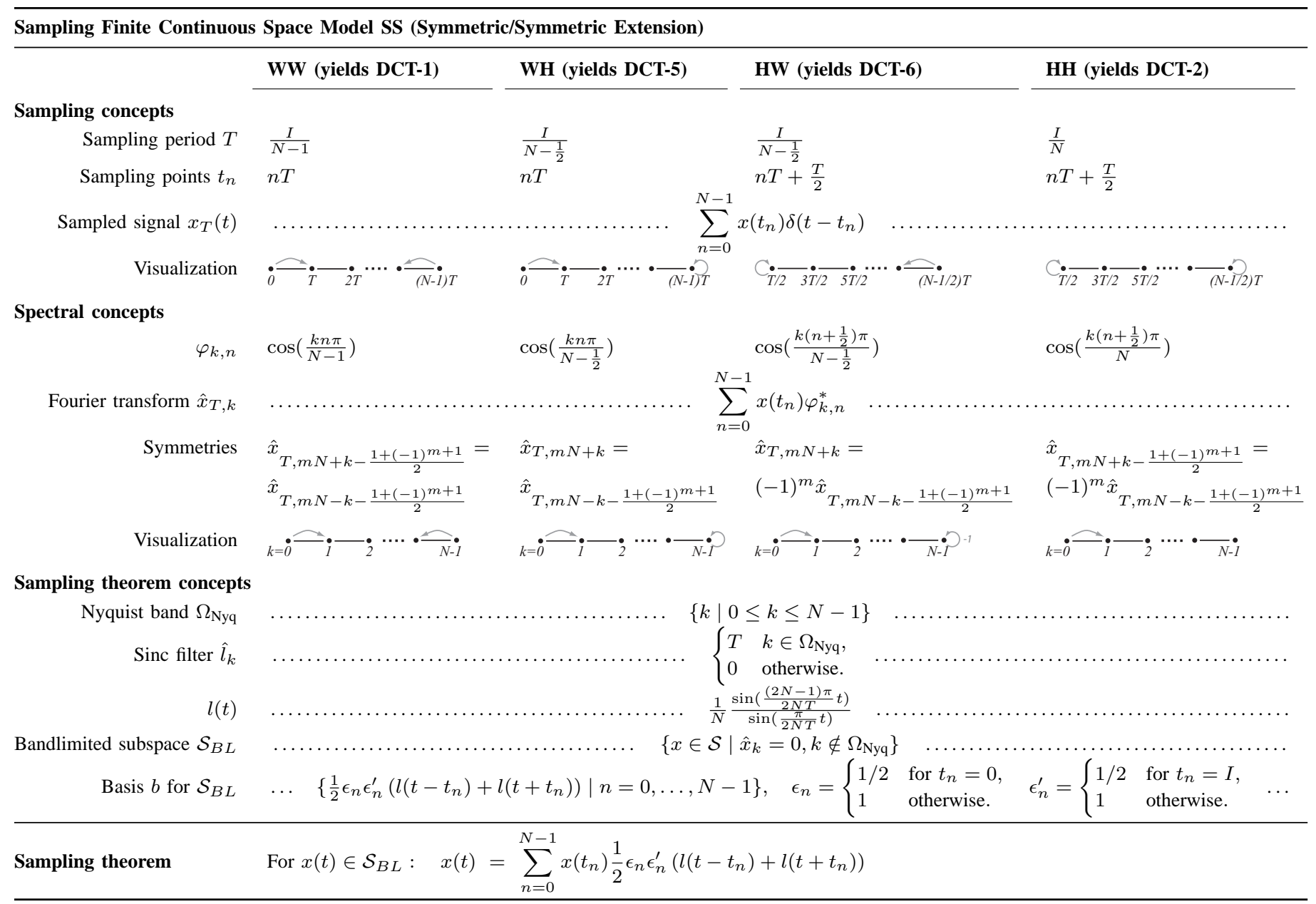

which can be reconstructed from their sampled versions, is $\mathcal{S}_{B L}=\left\{x \in \mathcal{S} \mid \hat{x}_{k}=0, k \notin \Omega_{\mathrm{Nyq}}\right\}$.

\section{B. Sampling Finite Continuous Space}

For each of the four finite space models in Table VII we have four choices of equidistant sampling, which arise from the treatment of the two boundaries (each one is either $S$ or A). Namely, for an S boundary we have the two choices W and $\mathrm{H}$ shown in Fig. 3 and for an A boundary the two choices $\mathrm{W}$ and $\mathrm{H}$ shown in Fig. 4. This means that each of the four finite continuous models will spawn four sampled versions (WW, WH, HW, HH) for a total of sixteen finite discrete space models. As we will show, these have exactly the sixteen DCTs/DSTs as their corresponding Fourier transforms.

We derive in detail the case of HH sampling the SS model (Table VII), which will yield the most widely used DCT of type 2. Then we briefly discuss the other three cases of sampling SS and collect the results in Table IX. Finally, we discuss the sampling of the other finite space models.

Select the shift. We fix a space shift by $T$.

Sample the signal and compute Fourier transform. We sample $\mathrm{HH}$, that is, the first sampling point is $T / 2$ and the last one is $I-T / 2$. To get $N$ samples, $T=I / N$ and $t_{n}=$ $n T+T / 2, n=0, \ldots, N-1$. The sampled signal is

$$
x_{T}(t)=\sum_{n=0}^{N-1} x\left(t_{n}\right) \delta\left(t-t_{n}\right),
$$

and applying the Fourier transform from Table VII, column SS, we get

$$
\hat{x}_{T, k}=\sum_{n=0}^{N-1} x\left(t_{n}\right) \cos \left(\frac{k\left(n+\frac{1}{2}\right) \pi}{N}\right),
$$

which, with $x_{n}=x(n T+T / 2)$, is nothing else but the DCT, type 2 , of $x_{0}, \ldots, x_{N-1}$. After sampling we obtain the graph structure shown under "Visualization." This shows that the DCT, type 2, is a Fourier transform for the finite space model with this visualization, that is, $\mathrm{HH}$ extensions on both sides.

The adjacency matrix of the visualization is indeed diagonalized by the DCT, type 2, [6], which confirms the correctness of our construction.

Find the symmetries in the Fourier transform. The sampled signal spectrum in (37) has the following symmetry properties: $\hat{x}_{T,-k}=\hat{x}_{T, k}$ on the left, and $\hat{x}_{T, N}=0$, $\hat{x}_{T, N+k}=-\hat{x}_{T, N-k}$ on the right. Taken together, for $n \in \mathbb{Z}$,

$$
\hat{x}_{T, m N+k-\frac{1+(-1)^{m+1}}{2}}=(-1)^{m} \hat{x}_{T, m N-k-\frac{1+(-1)^{m+1}}{2}},
$$


TABLE X

ESSENTIAL CONCEPTS FOR SAMPLING FINITE CONTINUOUS SPACE MODEL WITH EXTENSION SA.

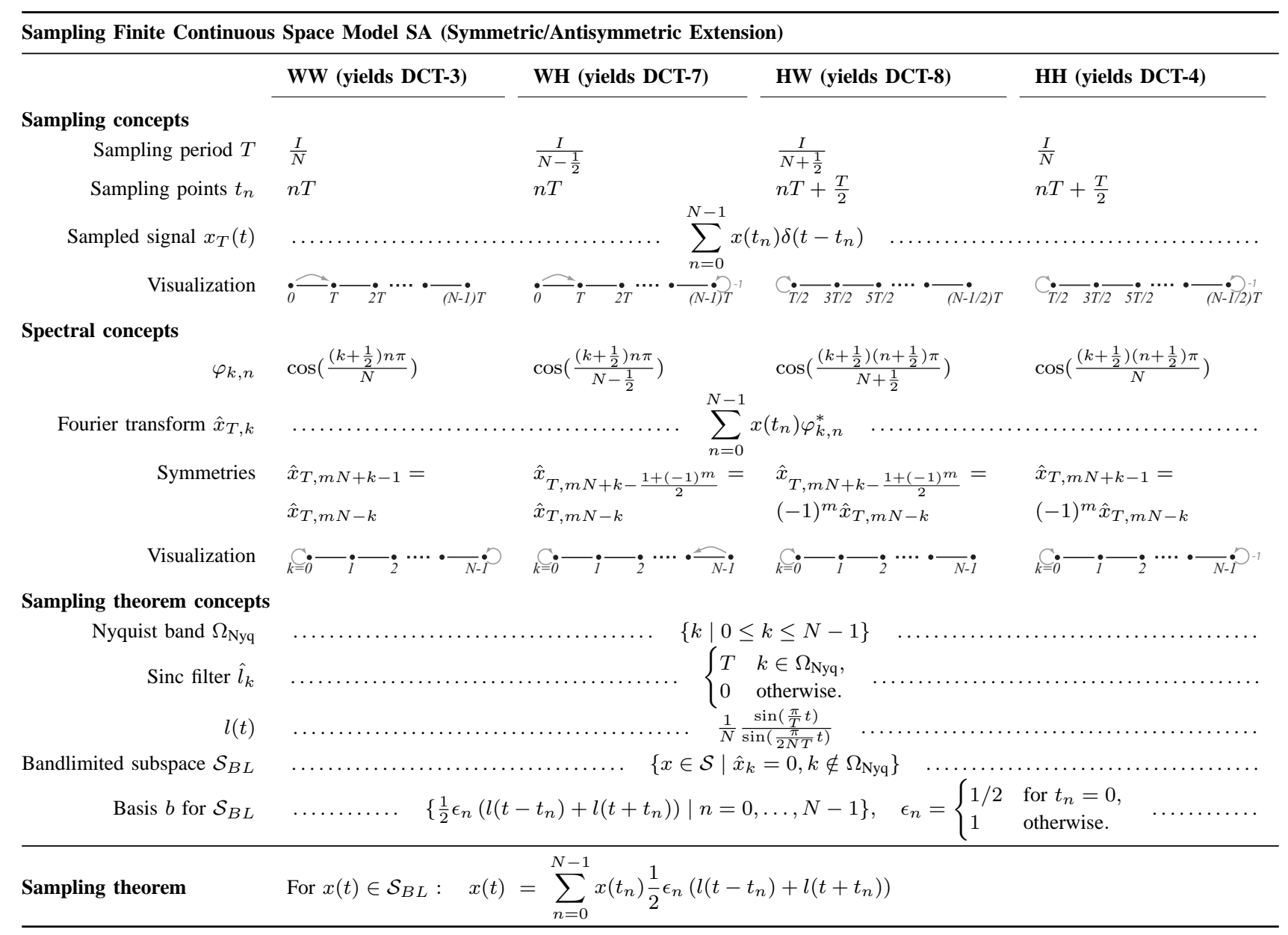

which yields the spectrum structure shown in Table IX, under the heading HH. Note that in our visualization of the spectrum, there is no loop of weight -1 on the right side; this is because the value of $\hat{x}_{T, k}$ at $k=N$ is 0 , and hence the node vanishes. The Nyquist band is again $\Omega_{\mathrm{Nyq}}=\{k \mid 0 \leq k \leq N-1\}$.

Extract the original spectrum. Similarly to what we did in the time case, and again assuming that the signal is bandlimited to the Nyquist band, we extract only that band by the suitable box and compute the inverse frequency response using its definition in Table VII, column SS.

$$
\hat{l}_{k}=\left\{\begin{array}{ll}
T & k \in \Omega_{\mathrm{Nyq}}, \\
0 & \text { otherwise. }
\end{array}, \quad l(t)=\frac{1}{N} \frac{\sin \left(\frac{(2 N-1) \pi}{2 N T} t\right)}{\sin \left(\frac{\pi}{2 N T} t\right)} .\right.
$$

Sampling theorem. As before, the extraction process which results in the original signal, is pointwise multiplication of the sampled signal $\hat{x}_{T, k}$ and the sinc filter $\hat{l}_{k}$ in the Fourier domain, or, equivalently, convolution (from Table VII, column $\mathrm{SS}$ ) of the sampled signal by the inverse frequency response of the filter in the original domain $l(t) *_{S S} x_{T}(t)$.

Theorem 5 (Sampling theorem: FS Model-SS-HH) For a signal $x(t)$ bandlimited to the Nyquist band:

$$
x(t)=\sum_{n=0}^{N-1} x\left(t_{n}\right) \frac{1}{2}\left(l\left(t-t_{n}\right)+l\left(t+t_{n}\right)\right) .
$$

As always, the space $\mathcal{S}_{B L}$ of bandlimited signals consists of those whose spectral support is limited to $\Omega_{\mathrm{Nyq}}$. The sampling theorem reveals the basis for this space. As expected, the basis functions are perfect interpolators as we show in the Appendix for this case.

Sampling SS: WW, WH, HW. Since the underlying continuous model is the same, the sinc takes the same form in all cases. The only difference to HH sampling is that the sampling locations include one (WH and $\mathrm{HW}$ ) or two (WW) boundary points, which affects $l(t) * x_{T}(t)$ due to (26). We accommodate this in Table IX by introducing the correction factors $\epsilon_{n}, \epsilon_{n}^{\prime}$.

Sampling SA, AS, AA. S boundaries are sampled as before ( $\mathrm{H}$ or $\mathrm{W}$; see Fig. 3), and A boundaries as shown in Fig. 4, that is, the boundary point is always omitted since the signal value is know to be zero.

The AA model shares the frequency response (and its inverse) with the SS model; hence the sinc is the same as for SS.

Similarly, the SA and AS model share the sinc, which now takes a different form, computed, as usual, as the inverse frequency response of the box:

$$
\hat{l}_{k}=\left\{\begin{array}{ll}
T & k \in \Omega_{\text {Nyq }}, \\
0 & \text { otherwise. }
\end{array}, \quad l(t)=\frac{1}{N} \frac{\sin \left(\frac{\pi}{T} t\right)}{\sin \left(\frac{\pi}{2 N T} t\right)} .\right.
$$


TABLE XI

ESSENTIAL CONCEPTS FOR SAMPLING FINITE CONTINUOUS SPACE MODEL WITH EXTENSION AS.

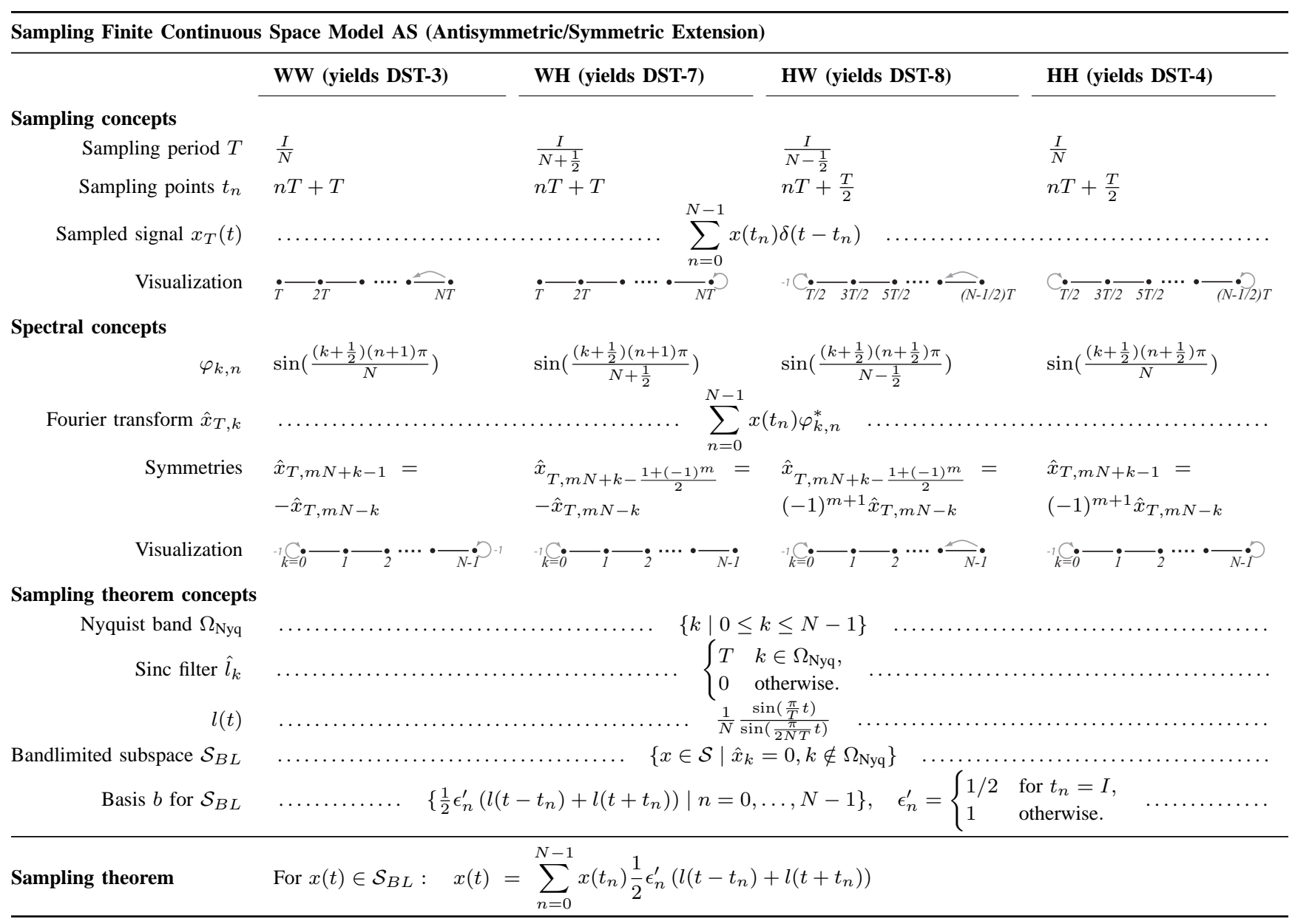

All other concepts are shared with the SS case. The results are summarized in Tables IX-XII.

Finally, we note that the tables reveal which DCTs and DSTs are (close to be) inverses of each other. To find the inverse for a given DCT or DST, we first look up the visualization of its spectral domain and then retrieve the transform that has the same visualization for its space domain. For example, the spectral domain visualization associated with the DCT-2 in Table IX corresponds to the space domain visualization associated with the DCT-3 in Table X and vice-versa.

To obtain the exact relationships, more tools are needed [12], [6].

\section{CONCLUSIONS}

In this paper we developed the complete continuous signal processing framework, called space signal processing, which underlies the sixteen discrete cosine and sine transform, and, in doing so, a novel way of deriving them through sampling. To organize the framework in exactly the same way as standard time signal processing, several key insights were needed. First, the realization that a different shift operation, namely the symmetric space shift, was needed as starting point. Second, a viewpoint that replaces signals with symmetries (for example, periodic) by signals on a compact domain with structure (for example, a circle). Third, the rigorous decoupling of filter space and signal space, multiplication of filters and filtering, and of Fourier transform and frequency response. All these are naturally provided by the algebraic signal processing theory of which this paper is an application.

This paper complements our prior, bottom-up derivation of the finite space models in [6]. The latter had the advantage that it also revealed the " $z$-transforms" and the polynomial algebras associated with the DCTs/DSTs. These algebras are the key to deriving their fast algorithms [26]. Together with this paper, [6], [26] provide a complete linear, nonstochastic theory of the DCTs/DSTs, which also may prove amenable to teaching this material.

\section{APPENDIX}

Let $l(t)$ be as defined in (38), and let $t_{n}=n T+T / 2$, $0 \leq n<N$. We show that $\left\{r_{n}(t)=\left(l\left(t-t_{n}\right)+l\left(t+t_{n}\right)\right) / 2 \mid\right.$ $0 \leq n<N\}$ are perfect interpolators, that is, $r_{n}\left(t_{m}\right)=0$ for $n \neq m$ and $r_{n}\left(t_{n}\right)=1$.

As the first set we compute $l(p T)$, for $p \in \mathbb{Z},|p|<2 N$. If $p=0$, L'Hôpital's rule yields $l(p T)=l(0)=(2 N-1) / N$. For $p \neq 0$,

$$
l(p T)=\frac{1}{N} \frac{\sin \left(p \pi-\frac{p}{2 N} \pi\right)}{\sin \frac{p}{2 N} \pi}=(-1)^{p+1} \frac{1}{N} .
$$


TABLE XII

ESSENTIAL CONCEPTS FOR SAMPLING FINITE CONTINUOUS SPACE MODEL WITH EXTENSION AA.

Sampling Finite Continuous Space Model AA (Antisymmetric/Antisymmetric Extension)

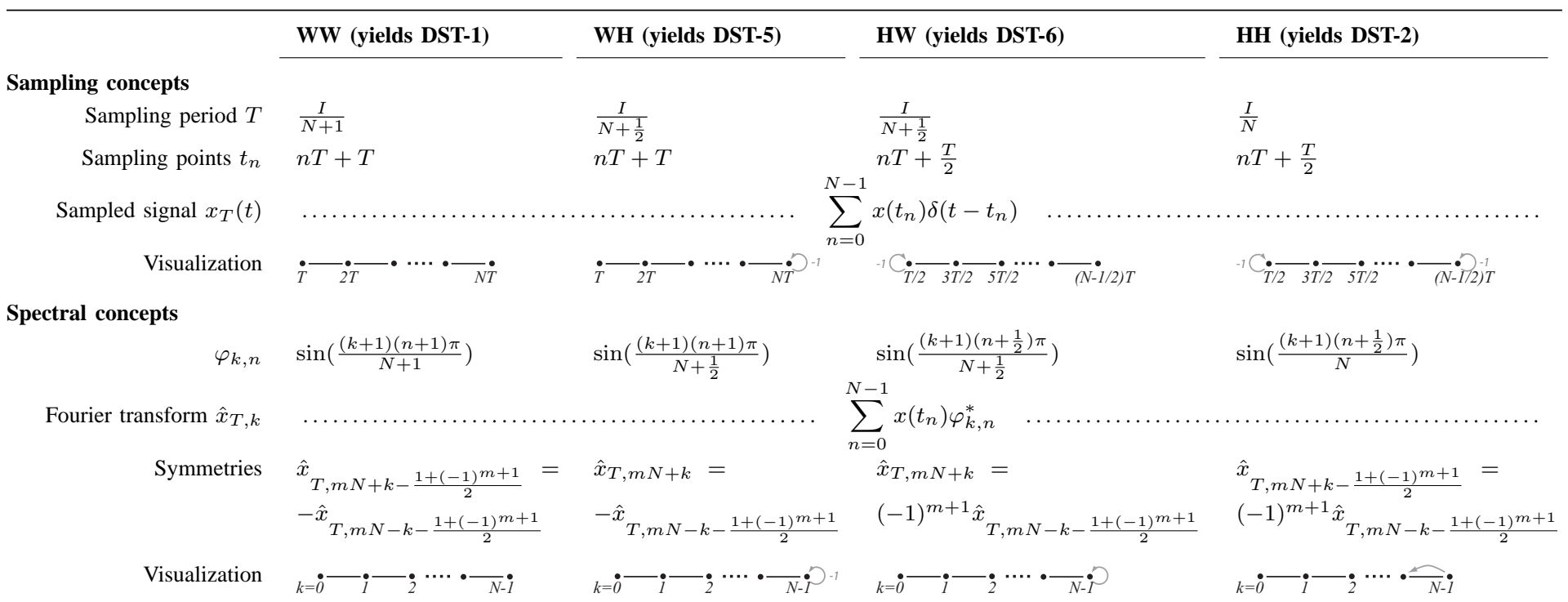

Sampling theorem concepts

Nyquist band $\Omega_{\mathrm{Nyq}}$

$\{k \mid 0 \leq k \leq N-1\}$

Sinc filter $\hat{l}_{k}$

$\begin{cases}T & k \in \Omega_{\mathrm{Nyq}} \\ 0 & \text { otherwise. }\end{cases}$

$l(t)$

$\frac{1}{N} \frac{\sin \left(\frac{(2 N-1) \pi}{2 N T} t\right)}{\sin \left(\frac{\pi}{2 N T} t\right)}$

Bandlimited subspace $\mathcal{S}_{B L}$

Basis $b$ for $\mathcal{S}_{B L}$

$$
\left\{x \in \mathcal{S} \mid \hat{x}_{k}=0, k \notin \Omega_{\mathrm{Nyq}}\right\}
$$

Sampling theorem $\quad$ For $x(t) \in \mathcal{S}_{B L}: \quad x(t)=\sum_{n=0}^{N-1} x\left(t_{n}\right) \frac{1}{2}\left(l\left(t-t_{n}\right)+l\left(t+t_{n}\right)\right)$

Now we can compute $r_{n}\left(t_{m}\right)=(l((m-n) T)+l((m+n+$ 1) $T) / 2$ assuming $0 \leq n, m<N$. For $n=m$,

$$
r_{n}\left(t_{n}\right)=\frac{1}{2}\left(l(0)+l((2 n+1) T)=\frac{1}{2}\left(\frac{2 N-1}{N}+\frac{1}{N}\right)=1 .\right.
$$

For $n \neq m$,

$$
r_{n}\left(t_{n}\right)=\frac{1}{2}\left(\frac{(-1)^{m-n}}{N}+\frac{(-1)^{m+n+1}}{N}\right)=0
$$

as desired.

\section{REFERENCES}

[1] J. Kovačević and M. Püschel, "Sampling theorems for trigonometric transforms," in Proc. IEEE Int. Conf. Acoust., Speech and Signal Proc., Toulouse, France, May 2006, vol. III, pp. 357-360.

[2] N. Ahmed, T. Natarajan, and K. R. Rao, "Discrete cosine transform," IEEE Trans. on Computers, vol. C-23, pp. 90-93, 1974.

[3] K. R. Rao and P. Yip, Discrete Cosine Transform: Algorithms, Advantages, Applications, Academic Press, 1990.

[4] M. Püschel and J. M. F. Moura, "Algebraic signal processing theory: Foundation and 1-D time," IEEE Trans. on Signal Processing, vol. 56, no. 8, pp. 3572-3585, 2008.

[5] M. Püschel and J. M. F. Moura, "Algebraic signal processing theory," [Online]. Available: http://arxiv.org/abs/cs.IT/0612077.

[6] M. Püschel and J. M. F. Moura, "Algebraic signal processing theory: 1-D space," IEEE Trans. on Signal Processing, vol. 56, no. 8, pp. 35863599, 2008.

[7] M. Püschel and M. Rötteler, "Algebraic signal processing theory: 2-D hexagonal spatial lattice," IEEE Trans. on Image Processing, vol. 16, no. 6, pp. 1506-1521, 2007.
[8] M. Püschel and M. Rötteler, "Fourier transform for the spatial quincunx lattice," in Proc. International Conference on Image Processing (ICIP), 2005, vol. 2, pp. 494-497.

[9] R. E. Edwards, Fourier Series: A Modern Introduction, vol. I, Holt, Rinehart and Winston, 1967.

[10] J. S. Walker, Fourier Analysis, Oxford University Press, 1988.

[11] S. A. Martucci, "Symmetric convolution and the discrete sine and cosine transforms," IEEE Trans. on Signal Processing, vol. 42, no. 5, pp. 10381051, 1994.

[12] G. Strang, "The discrete cosine transform," SIAM Review, vol. 41, no. 1, pp. 135-147, 1999.

[13] Z. Wang, "Interpolation using type I discrete cosine transform," Electronic Letters, vol. 26, pp. 1170-1172, 1990.

[14] S. Martucci, "Interpolation in the DST and DCT domains," in Proceedings International Conference on Image Processing (ICIP), 2000, vol. 2, pp. 339-342.

[15] H. Park, Y. Park, and Oh S.-K., "L/M-fold image resizing in blockDCT domain using symmetric convolution," IEEE Trans. on Image Processing, vol. 12, no. 9, pp. 1016-1034, 2003.

[16] Atoyan A. and Patera J., "Properties of continuous Fourier extension of the discrete cosine transform and its multidimensional generalization," J. Math. Phys., vol. 45, no. 6, pp. 2468-2491, 2004.

[17] M. Unser, "Sampling-50 years after Shannon," Proc. IEEE, vol. 88, no. 4, pp. 569-587, Apr. 2000.

[18] W. C. Curtis and I. Reiner, Representation Theory of Finite Groups, Interscience, 1962.

[19] Paul A. Fuhrman, A Polynomial Approach to Linear Algebra, Springer Verlag, New York, 1996.

[20] C. E. Shannon, "Communications in the presence of noise," Proc. IRE, vol. 37, pp. 10-21, 1949.

[21] J. M. Whittaker, "The Fourier theory of the cardinal functions," in Proc. Math. Soc. Edinburgh, 1929, vol. 1, pp. 169-176. 
[22] V. A. Kotelnikov, "On the transmission capacity of ether and wire in electrocommunications," Izd. Red. Upr. Svyazzi RKKA, 1933.

[23] H. Nyquist, "Certain topics in telegraph transmission theory," Trans. AIEE, vol. 47, pp. 617-644, Apr. 1928.

[24] A. V. Oppenheim and R. W. Schafer, Discrete-time Signal Processing, Prentice Hall, Englewood Cliffs, NJ, 1989.

[25] M. Vetterli and J. Kovačević, Wavelets and Subband Coding, Signal Processing. Prentice Hall, Englewood Cliffs, NJ, 1995, http://waveletsandsubbandcoding.org/.

[26] M. Püschel and J. M. F. Moura, "Algebraic signal processing theory: Cooley-Tukey type algorithms for DCTs and DSTs," IEEE Trans. on Signal Processing, vol. 56, no. 4, pp. 1502-1521, 2008. 